\title{
Mouse fukutin deletion impairs dystroglycan processing and recapitulates muscular dystrophy
}

\author{
Aaron M. Beedle, ${ }^{1,2}$ Amy J. Turner, ${ }^{1}$ Yoshiaki Saito, ${ }^{1}$ John D. Lueck, ${ }^{1}$ Steven J. Foltz, ${ }^{2}$ \\ Marisa J. Fortunato, ${ }^{2}$ Patricia M. Nienaber, ${ }^{1}$ and Kevin P. Campbell ${ }^{1}$ \\ ${ }^{1}$ Howard Hughes Medical Institute, Departments of Molecular Physiology and Biophysics, Neurology and Internal Medicine, Carver College of Medicine, \\ University of lowa, lowa City, lowa, USA. ${ }^{2}$ Department of Pharmaceutical and Biomedical Sciences, College of Pharmacy, \\ University of Georgia, Athens, Georgia, USA.
}

\begin{abstract}
Dystroglycan is a transmembrane glycoprotein that links the extracellular basement membrane to cytoplasmic dystrophin. Disruption of the extensive carbohydrate structure normally present on $\alpha$-dystroglycan causes an array of congenital and limb girdle muscular dystrophies known as dystroglycanopathies. The essential role of dystroglycan in development has hampered elucidation of the mechanisms underlying dystroglycanopathies. Here, we developed a dystroglycanopathy mouse model using inducible or muscle-specific promoters to conditionally disrupt fukutin (Fktn), a gene required for dystroglycan processing. In conditional Fktn-KO mice, we observed a near absence of functionally glycosylated dystroglycan within 18 days of gene deletion. Twentyweek-old KO mice showed clear dystrophic histopathology and a defect in glycosylation near the dystroglycan $O$-mannose phosphate, whether onset of Fktn excision driven by muscle-specific promoters occurred at E8 or E17. However, the earlier gene deletion resulted in more severe phenotypes, with a faster onset of damage and weakness, reduced weight and viability, and regenerating fibers of smaller size. The dependence of phenotype severity on the developmental timing of muscle Fktn deletion supports a role for dystroglycan in muscle development or differentiation. Moreover, given that this conditional Fktn-KO mouse allows the generation of tissue- and timingspecific defects in dystroglycan glycosylation, avoids embryonic lethality, and produces a phenotype resembling patient pathology, it is a promising new model for the study of secondary dystroglycanopathy.
\end{abstract}

\section{Introduction}

The dystrophin-glycoprotein complex (DGC) links the intracellular cytoskeleton to the extracellular basement membrane and provides a scaffold for associated proteins to promote tissue structural integrity and cell signaling $(1,2)$. Disruption of the DGC, via mutations in dystrophin, or the transmembrane sarcoglycans results in a loss of or reduction in the complex at the sarcolemma and a subsequent membrane fragility, causing muscular dystrophies of moderate to mild phenotypes (dystrophin: Duchenne and Becker muscular dystrophies; $\gamma, \alpha, \delta$ sarcoglycans: limb girdle muscular dystrophies [LGMDs] type 2C, 2D, 2E, and 2F) (3). More recently, a new class of diseases, called secondary dystroglycanopathies, has been identified. Dystroglycan, encoded by DAG1, is a DGC protein with an extracellular $\alpha$ subunit and a transmembrane $\beta$ subunit (4). In secondary dystroglycanopathies, direct mutations are not present in dystroglycan itself; rather, part of an extensive and complex carbohydrate structure is lost from the extracellular surface of the $\alpha$ DG subunit (5). This glycan serves as the epitope for $\alpha \mathrm{DG}$ binding to laminin, agrin, perlecan, and neurexin. Therefore, glycosylation deficiency severs the DGC link to the extracellular matrix.

Mutations in 6 genes encoding known or putative glycosyltransferases cause a failure in $\alpha \mathrm{DG}$ glycosylation: protein $O$-mannosyltransferase 1 and 2 (POMT1/2, refs. 6, 7); protein $O$-mannose $\beta-1$,

Conflict of interest: Kevin P. Campbell is a scientific advisory board member and consultant for ARMGO Pharma Inc. He also has a patent on increasing the functional glycosylation of $\alpha$-dystroglycan in the treatment of muscle degeneration. Citation for this article: J Clin Invest. 2012;122(9):3330-3342. doi:10.1172/JCI63004
2-N-acetylglucosaminyltransferase (POMGNT1, ref. 8); fukutin (FKTN, ref. 9); FKTN-related protein (FKRP, ref. 10); and glycosyltransferase-like protein (LARGE, ref. 11). Collectively, these genes can cause severe congenital or milder limb girdle muscular dystrophies: Walker Warburg syndrome (WWS, various); muscle eye brain disease (MEB, various); Fukuyama congenital muscular dystrophy (Fukuyama congenital muscular dystrophy [FCMD], FKTN); congenital muscular dystrophy type 1C (MDC 1C, FKRP); and limb girdle muscular dystrophy (LGMD) types 2I (FKRP), 2K (POMT1), 2M (FKTN), 2N (POMT2), and MDDGC3 (POMGNT1). POMT1/ POMT2 and POMGnT1 have known activities in the synthesis of a rare $O$-mannose tetrasaccharide on dystroglycan $(8,12-14)$. Recently, an intriguing $O$-mannose phosphate linkage was found on dystroglycan and was identified as a putative site of action for LARGE (15), a xylosyltransferase and glucuronyltransferase (14). The specific functions of FKTN and FKRP are unknown; however, both are believed to contribute to the $O$-mannose glycan.

Study of dystroglycanopathy disease mechanisms and $\alpha \mathrm{DG}$ glycosylation is hampered by the essential nature of dystroglycan in development. Complete $\mathrm{KO}$ of dystroglycan or its processing genes, with the exception of Pomgnt1, causes embryonic lethality in mice (16-20). Furthermore, attempts to generate disease models by knocking in patient mutations have generally been unsuccessful because (a) residual $\alpha$ DG processing activity is sufficient to prevent the development of dystrophic disease (21), and (b) many mutant mice die within 1 or 2 days of birth $(20,22)$. To date, the most commonly used dystroglycanopathy model is the myodystrophy mouse (Large $e^{m y d} /$ myd $)$, which harbors a spontaneous mutation in Large (11). Large $e^{\text {myd/myd }}$ mice exhibit muscle and brain pheno- 
types, loss of functional $\alpha$ DG glycosylation, and reduced lifespan. Although this is a very useful model, the presence of severe brain abnormalities impairs mouse growth and movement and can hinder more in-depth studies of the muscle disease.

To better understand disease mechanisms and characterize $\alpha$ DG hypoglycosylation in dystroglycanopathies, we sought to develop a mouse model for conditional disruption of $\alpha \mathrm{DG}$ processing. We targeted the dystroglycanopathy gene Fktn, encoding fukutin, because it is well established to cause both severe and milder muscular dystrophies, its complete gene $\mathrm{KO}$ is embryonic lethal in mice (23), and an animal model may be critical to discovering its biological function. Using Cre/LoxP and Flp/Frt technologies $(24,25)$, we established a mouse line for conditional disruption of Fktn. Using muscle-specific promoters (myf5 and muscle creatine kinase $[\mathrm{MCK}]$ ), we generated dystroglycanopathy mice with severe and milder symptoms that are representative of the spectrum of patient diseases. We found evidence that Fktn disruption during muscle development at E8 causes a more severe phenotype than Fktn $\mathrm{KO}$ initiated at $\mathrm{E} 17$ in differentiating muscle and discovered that FKTN activity is necessary at or near $\alpha$ DG's unusual $O$-mannose phosphate linkage. These studies validate a new mouse model for dystroglycanopathy mechanistic and therapeutic discovery.

\section{Results}

To develop a model for dystroglycanopathy muscular dystrophies, we created a mouse line with LoxP sites flanking Fktn exon 2 for conditional deletion of the Fktn start codon. Both Fktn "null" (Cre-recombined) and floxed Fktn (neo cassette removed) alleles were generated using Cre/LoxP or Flp/Frt germline recombination, respectively (Supplemental Figure 1; supplemental material available online with this article; doi:10.1172/JCI63004DS1). Consistent with previous reports, we could not obtain complete KO mice, confirming embryonic lethality of germline Fktn disruption (Supplemental Figure 1D and refs. 17, 23). Surprisingly, the incidence of homozygosity for the floxed Fktn allele was also subMendelian, indicating that some lethality is associated with floxed exon 2 even in the absence of Cre recombinase (Supplemental Figure 1D). Fktn ${ }^{f / f l}$ mice that survived to birth were indistinguishable from wild-type littermates.

Whole-animal Fktn disruption impairs $\alpha D G$ glycosylation. To validate Cre recombination at the Fktn locus and confirm disruption of $\alpha D G$ glycosylation in vivo, we used whole-animal tamoxifeninducible (Tam-inducible) Cre mice ( $\mathrm{Tg}^{\mathrm{Cre}-E s s 1}$; The Jackson Laboratory, no. 004682) to induce Fktn exon 2 deletion. Tg ${ }^{\mathrm{Cre}-\mathrm{Esr1}} ; \mathrm{Fktn} \mathrm{fl}^{\mathrm{l} / \text { - }}$ mice (iKO) and littermates were administered Tam or vehicle (Veh) at 6 weeks of age. Tam-treated iKO (Tam iKO) mice showed no difference in body weight, forelimb grip strength, or open-field activity compared with littermates from 4 through 20 weeks of age (data not shown). In contrast, serum creatine kinase (CK) levels in Tam iKO mice began to rise at 14 weeks, and were significantly higher than in control mice at 16 and 20 weeks of age, indicating muscle damage (Figure 1C and data not shown). Histological analysis of the iliopsoas muscle at 20 weeks confirmed the presence of dystrophic disease, with variation in fiber size, necrosis, and a significant increase in centrally nucleated fibers (Figure 1, A and B), indicative of muscle regeneration.

Staining for the $\alpha \mathrm{DG}$ glyco-epitope was nearly negative or patchy, while $\beta D G$ remained at the sarcolemma of Tam iKO mice, indicating a disruption in $\alpha \mathrm{DG}$ processing rather than abnormal protein expression (Figure 1A). This was confirmed by Western blotting with an antibody directed to the $\alpha$ DG core protein; $\alpha$ DG protein was present with a reduced molecular weight in Tam iKO muscle (Figure 1D). $\alpha$ DG glycosylation was partially disrupted in some Veh iKO mice (Figure 1D); however, histological analysis of 2 Veh iKO mice in this group revealed no significant abnormalities (data not shown). We hypothesized that the reduced $\alpha$ DG molecular weight in these Veh iKO mice was due to exposure to Tam while mice were cohoused with Tam littermates, so we performed additional experiments on Veh iKO mice caged separately from Tam littermates for 7 days after the first Tam dose or iKO mice from cages receiving no treatment. $\alpha D G$ glycosylation was normal in these uninduced iKO samples (Supplemental Figure 2A, 10 weeks after vehicle iKO [age 16 weeks]; and 20-week-old iKO; data not shown), confirming that untreated mice can be exposed to Tam from treated cagemates.

To assess the time from induction of Fktn gene excision to loss of $\alpha$ DG glycosylation, we Tam treated additional iKO mice and collected muscle tissues for analysis at various times. While a small amount of normal $\alpha D G$ was detected in wheat germ agglutinin-enriched (WGA-enriched) skeletal muscle of mice at 2.5 weeks after Tam treatment, loss of the $\alpha$ DG functional glycan was virtually complete at all times tested $(2.5,4,6$, and 10 weeks after Tam treatment), indicating that most wild-type $\alpha$ DG protein is depleted within 2.5 weeks of Cre induction (Supplemental Figure 2, A and B). In iliopsoas muscles, dystrophic pathology was first detected 6 weeks after Tam treatment (Supplemental Figure $2 \mathrm{C}$ ). However, in this assay, glycosylated $\alpha$ DG was still detected, albeit at reduced intensity (Supplemental Figure 2C), suggesting that immunofluorescence detection overestimates the expression of glycosylated $\alpha$ DG.

In addition to skeletal muscle, we confirmed a loss of/reduction in the $\alpha$ DG glyco-epitope in all Tam iKO tissues tested, with a concomitant reduction in core protein molecular weight (Figure 1D). The extent of $\alpha D G$ glycosylation is tissue specific (26). Highmolecular-weight (skeletal muscle, heart, kidney) and moderatemolecular-weight (brain, peripheral nerve, thymus) $\alpha$ DG glycans were affected by Fktn disruption, indicating that FKTN-dependent modifications take place in these tissues. For tissues with lowmolecular-weight $\alpha \mathrm{DG}$ (liver, lung, testes), there was a clear shift in liver and lung, and potentially one in testes, although in the latter case, this could have been an artifact of uneven sample loading. In general, our data are consistent with a recent report of $\alpha$ DG glycosylation in liver, lung, and testes by Kuga et al. (27). These data confirm that targeted disruption of Fktn exon 2 causes $\alpha$ DG glycosylation defects in vivo and support the use of this iKO model as a novel tool for the study of dystroglycanopathy disease mechanisms. Muscle-specific Fktn deletion disrupts the $\alpha D G$ functional binding glycan. To assess the importance of dystroglycan interactions with the extracellular matrix during muscle development and maintenance, we bred floxed Fktn mice to mice expressing Cre recombinase under the control of different muscle-specific promoters. The myogenic determination factor promoter myf5 directs Cre translation specifically in early skeletal muscle development and proliferation (E8-E14) and in satellite cells initiating muscle regeneration (28). This generates a complete muscle $\mathrm{KO}$, as Cre is expressed during muscle cell specification. In contrast, the MCK promoter is turned on late in striated muscle differentiation. With respect to muscle development, this coincides with onset of MCK expression at E17, reaching a peak sustained expression by P10 (29). Notably, in mature $\mathrm{KO}$ muscle, regenerating fibers are normal because 
A
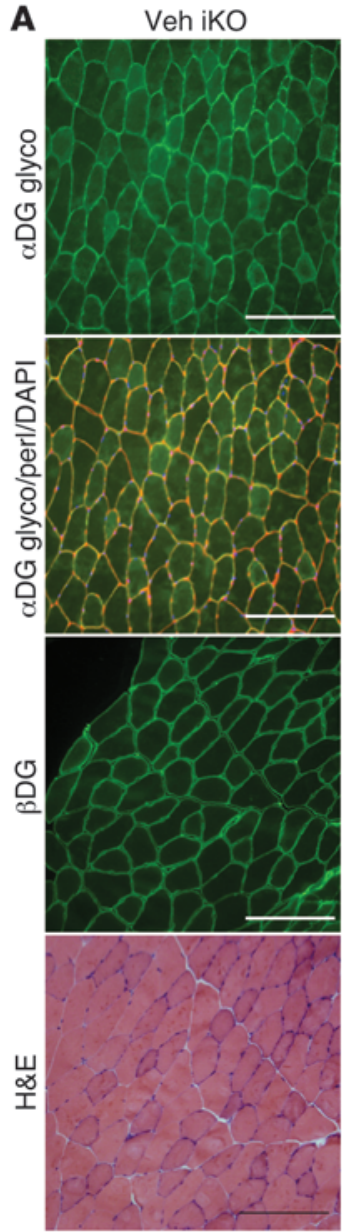

Tam iKO
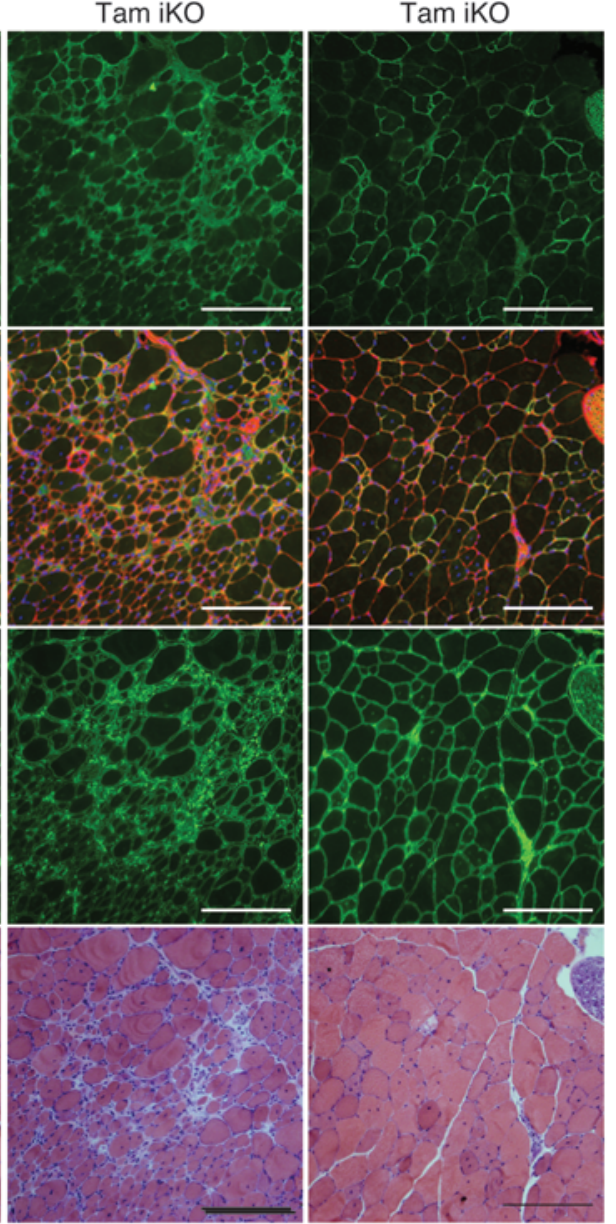

B
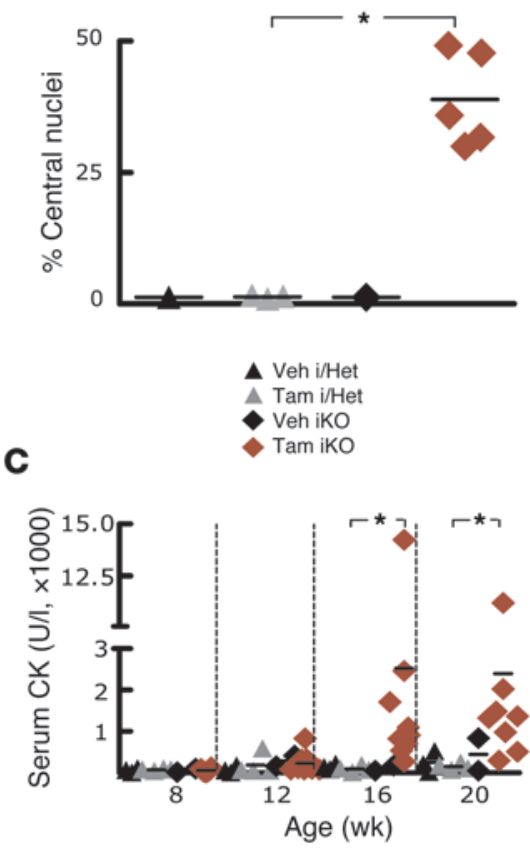

D
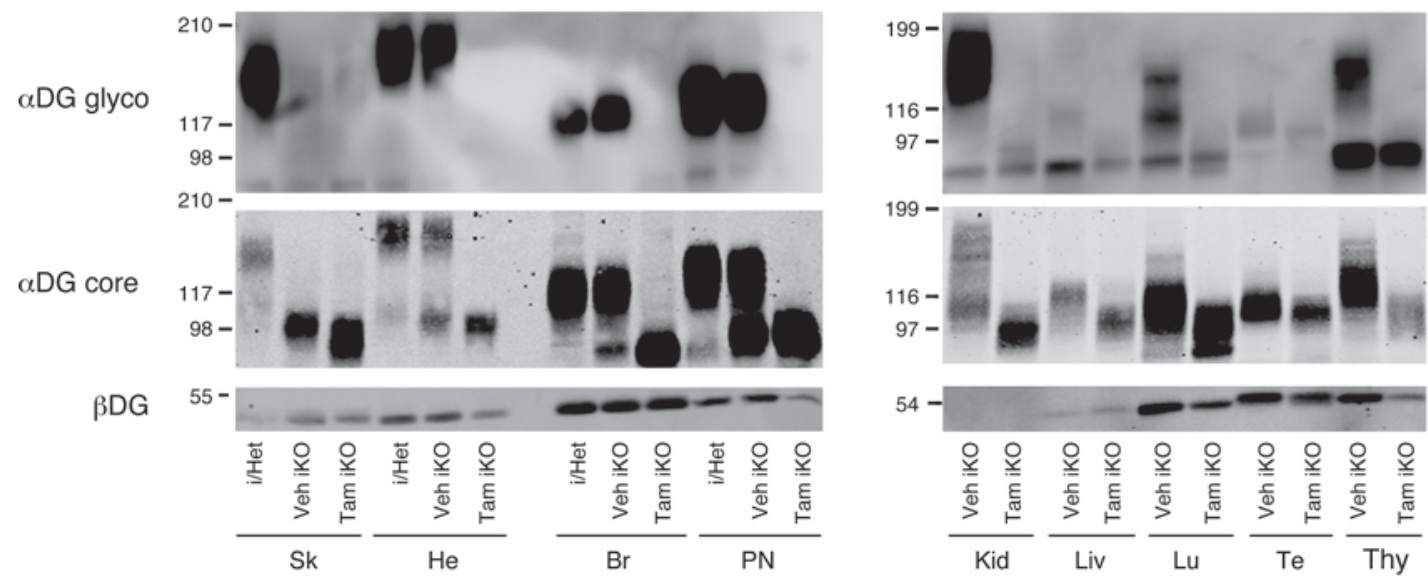

\section{Figure 1}

Inducible, whole-animal Fktn deletion causes dystrophic features with disruption of $\alpha$ DG glycosylation. (A) Representative iliopsoas images from

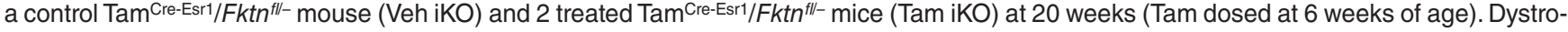
phic pathology is evident in Tam iKO muscle with H\&E staining. $\alpha$ DG glycosylation ( $\alpha$ DG glyco) is abnormal in KO mice, while $\beta D G$ is unchanged. ECM protein perlecan (perl) and nuclear (DAPI) counterstains are shown; original magnification, $\times 20$; scale bars: $100 \mu \mathrm{m}$. (B) The percentage of centrally nucleated fibers is plotted for individual mice. $\mathrm{i} / \mathrm{Het}$, heterozygous or inducible heterozygous. ${ }^{*} P=0.036$, Tam i/Het versus Tam iKO; Mann-Whitney test. (C) Serum CK activity is plotted for individual mice at various ages. ${ }^{\star} P \leq 0.05$; Dunn's test. (D) DG expression and glycosylation in various tissues. $\alpha \mathrm{DG}$ glyco detection by $\mathrm{IIH} 6$ is disrupted in all Tam iKO tissues tested, and core $\alpha \mathrm{DG}$ protein ( $\alpha \mathrm{DG}$ core) is reduced in mass. An intermediate phenotype was observed in some Veh iKO mice, indicating exposure to Tam from treated littermates. $\beta D G$ blots demonstrate different DG expression levels across tissues and confirm the presence of DG in Fktn-deficient mice. Sk, skeletal muscle; He, heart; Br, brain; PN, peripheral nerve; Kid, kidney; Liv, liver; Lu, lung; Te, testes; Thy, thymus. 
A

A Lam O/L
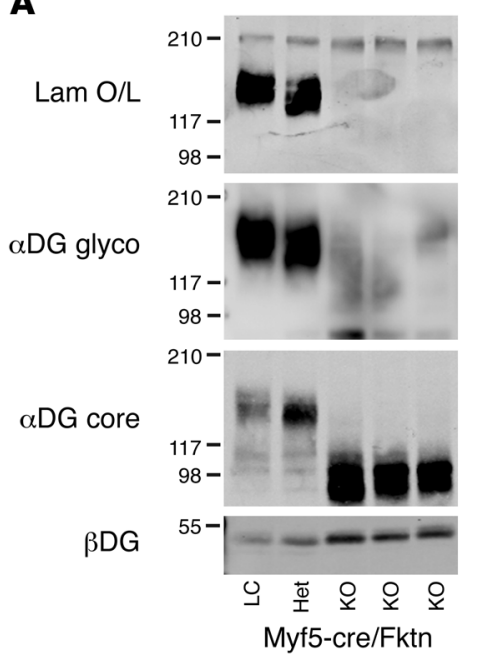

C

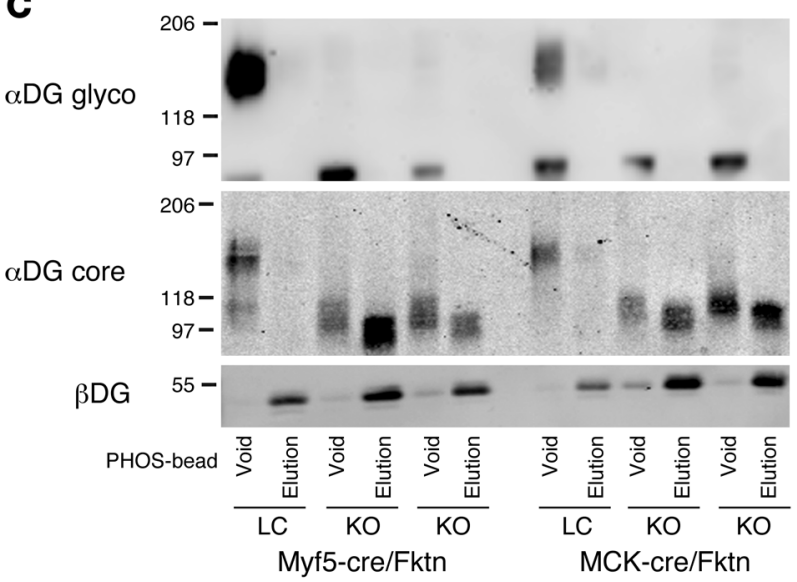

MCK is not expressed until these new fibers reach late differentiation. For simplicity, we will refer to gene excision in MCK-Cre/ Fktn mice as beginning at E17, but recognize that muscle regeneration is spared at any age.

WGA-enriched extracts of hind limb skeletal muscle from 20-week-old KO and littermate mice were analyzed for dystroglycan function and glycosylation. $\alpha$ DG from both myf5-Cre (E8) and MCK-Cre (E17) KOs failed to bind laminin in overlay assays, representing a loss of $\alpha \mathrm{DG}$ functional binding activity in musclespecific Fktn-deficient mice (Figure 2, A and B). Similarly, the $\alpha$ DG glycan epitope was dramatically reduced or absent in $\mathrm{KO}$ muscle (Figure 2, A and B). $\beta D G$ and $\alpha$ DG protein were present in all samples. However, $\alpha$ DG protein was only detected at a reduced molecular weight in $\mathrm{KO}$ muscle, confirming that $\alpha \mathrm{DG}$ glycosylation, not protein expression, was abnormal (Figure 2, A and B).

To assess the nature of the abnormal $\alpha \mathrm{DG}$ glycan in Fktn-KO mice, we concentrated WGA extracts for PHOS-bead experiments. PHOS-beads bind to exposed (free) phosphate groups on proteins or glycans under denaturing conditions. The recently discovered glyco-phosphate linkage on the $\alpha \mathrm{DG} O$-mannose is believed to be the branch that connects $\alpha$ DG's functional binding glycan (unknown structure) to the known $\alpha \mathrm{DG} O$-mannose. In the Large $e^{m y / m y d}$ dystroglycanopathy mouse, the glycan branch attached to the phosphate is missing, leaving a free phosphate group that

\section{Figure 2}

Biochemical analyses of dystroglycan in skeletal muscle from Fktn$\mathrm{KO}$ mice and littermates. (A) Western blot analyses of WGA-purified skeletal muscle from myf5-Cre/Fktn (E8) KO mice and littermates (LC, $\mathrm{Het}$ ). All KOs have reduced $\alpha \mathrm{DG}$ mass, as measured by detection of core protein ( $\alpha \mathrm{DG}$ core). This corresponds to a complete loss of $\alpha \mathrm{DG}$ binding activity in laminin overlay (Lam $\mathrm{O} / \mathrm{L}$ ) and loss or reduction of $\alpha D G$ glyco-epitopes ( $\alpha D G$ glyco). $\beta D G$ protein is detected in all cases. (B) Western blot analyses of MCK-Cre/Fktn (E17) KO and littermates, as in A. (C) Detection of $\alpha D G$ free phosphate. WGA-enriched skeletal muscle was applied to PHOS-beads to capture proteins with exposed phosphates. $\alpha \mathrm{DG}$ from control mice has no free phosphate, but E8 and E17 muscle KOs (myf5-Cre/Fktn KO, MCK-Cre/Fktn KO) contain a substantial proportion with exposed phosphate. $\beta D G$ is normally phosphorylated and serves as an experimental control.

binds to PHOS-beads (15). As shown in Figure 2C, normally glycosylated $\alpha \mathrm{DG}$ from littermate mice remained in the PHOS-bead void, indicating that there are no free phosphates on wild-type $\alpha D G$. In contrast, hypoglycosylated $\alpha \mathrm{DG}$ in myf5-Cre (E8) and MCK-Cre (E17) Fktn-KO mice distributes into two populations: an $\alpha D G$ species that remains in the void fraction; and a second species that binds to the PHOS-beads (Figure 2C). The $\alpha$ DG species in the PHOS-bead void and elution may constitute two distinct populations of $\alpha \mathrm{DG}-$ one with a free glyco-phosphate (elution) and one without (void).

Failure to bind the PHOS-beads could indicate that this KO $\alpha D G$ is missing the phosphate linkage, or that additional sugars (e.g., $X$ ) have been added to the phosphate so that it is no longer a free group (e.g., protein-O-mannose-P-X). To further examine these possibilities, we treated MCK-Cre (E17) KO muscle from the PHOS-bead void with aqueous hydrofluoric acid (HFaq). Cold HFaq specifically degrades phosphoester bonds and therefore will cleave any glycans attached to the phosphate group (e.g., protein$O$-mannose-P-X) (15). HFaq treatment of MCK-Cre (E17) KO $\alpha$ DG generated a small downward shift in molecular weight, indicating that the $\alpha D G$ fraction in the PHOS-bead void does contain the $O$-mannose phosphate linkage (i.e., protein-O-mannose-P-X; Supplemental Figure 3). Of interest, the HFaq-treated $\alpha D G$ in KO mice was slightly larger than that in wild-type mice, suggesting that some glycan structures, besides the $O$-mannose phosphate, are slightly larger on the $\alpha \mathrm{DG}$ of KO muscle, perhaps because adjacent glycans are accessible for further modification in the absence of the large laminin-binding glycan. These data provide further evidence that FKTN contributes to modifications made at or just downstream of the $O$-mannose phosphate in the synthesis of $\alpha D G$ 's laminin-binding glycan, but is not directly involved in phosphorylation process.

Loss of Fktn beginning at $E 17$ in differentiated striated muscle confers mild, progressive dystrophic changes in mice. We were surprised to find that $\alpha D G$ from 20-week-old mice of myf5-Cre/Fktn (E8) versus MCK-Cre/Fktn (E17) muscle KOs was virtually indistinguishable biochemically (see above). To determine whether the timing of $\alpha D G$ glycosylation disruption affected dystroglycanopathy disease development or progression, we undertook a comprehensive phenotypic study of both conditional KO strains. In agreement with the biochemical findings, MCK-Cre/Fktn (E17) KO mice had evidence of abnormal dystroglycan glycosylation, as the $\alpha D G$ functional glycan was reduced and patchy in KO mice compared with littermates, while the $\alpha D G$ and $\beta D G$ protein expres- 
A
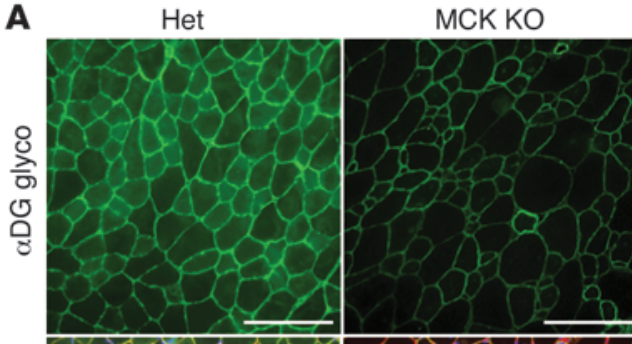

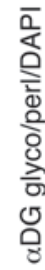

믐

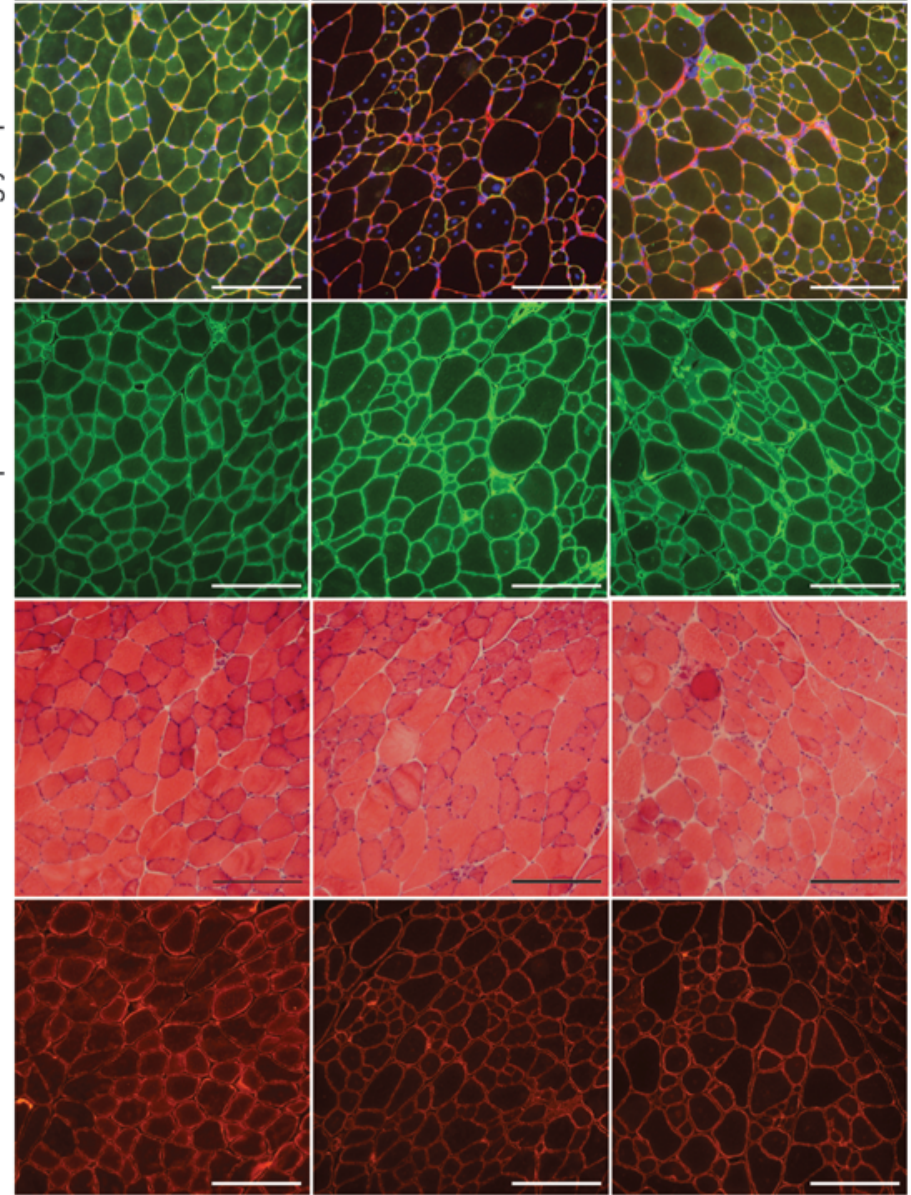

MCK KO

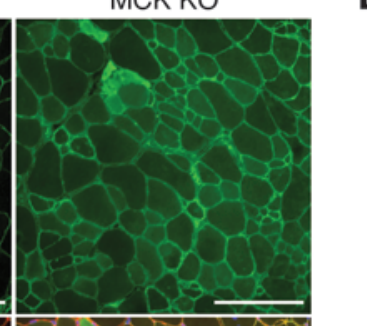

B

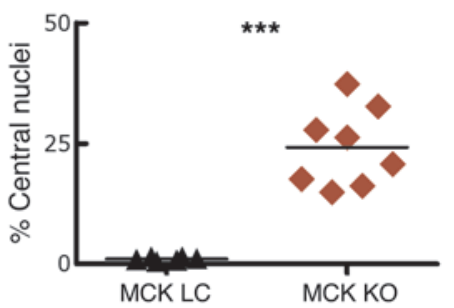

C

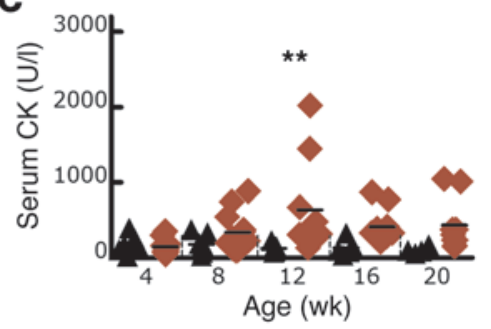

D

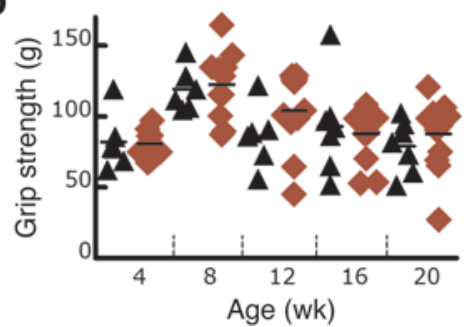

E
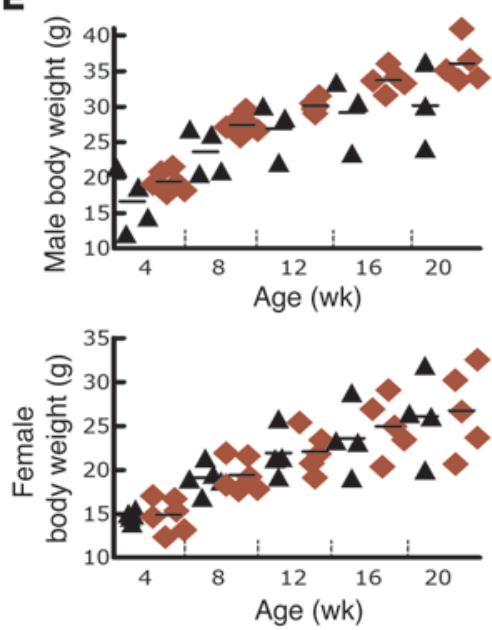

Figure 3

Cre recombinase driven at E17 by the MCK promoter directs Fktn deletion in differentiating striated muscle to cause phenotypes consistent with mild muscular dystrophy. (A) H\&E and immunofluorescence images from the iliopsoas of 20-week-old Het and KO mice. Dystrophic pathology is observed in $\mathrm{KO}$ mice along with patchy expression of glycosylated $\alpha \mathrm{DG}$ ( $\alpha \mathrm{DG}$ glyco). $\alpha \mathrm{DG}$ core protein and $\beta \mathrm{DG}$ are unchanged. DAPI nuclear stain and perlecan are shown for comparison; original magnification, $\times 20$; scale bars: $100 \mu \mathrm{m}$. (B) The percentage of iliopsoas fibers with central nucleation is shown for individual 20 -week-old mice. ${ }^{\star \star *} P=0.0007$; Mann Whitney test. (C) CK activity is detected in serum of individual littermate and $\mathrm{KO}$ mice at various ages. ${ }^{* *} P=0.001-0.01,12-$ week MCK LC versus KO; Bonferroni test. (D) Analysis of average forelimb grip strength for individual mice at various ages did not reveal muscle weakness in KO mice. $P>0.05$; ANOVA. (E) Body weights of male and female LC and KO mice from 4 through 20 weeks $(P>0.05$; ANOVA). Black triangles, MCK-Cre LC; red diamonds, MCK-Cre Fktn-KO. Statistics were calculated for $\mathrm{LC}$ versus $\mathrm{KO}$ mice at each age.

sion was unchanged (Figure 3A). Patchy expression is expected in this line, as regenerating fibers should have normal glycosylation. Histologically, all MCK-Cre/Fktn (E17) KO mice had signs of dystrophic disease, with central nucleation, variation in fiber size, and hypercontracted fibers (Figure 3, A and B). The presence of muscle damage was confirmed by elevated serum $\mathrm{CK}$ at 12 weeks of age (Figure 3C). In addition, a muscle phenotype was revealed when mice were stressed to exhaustion with a treadmill 
run. MCK-Cre/Fktn (E17) KO mice ran for shorter times than littermates (Supplemental Figure 4A), and exercise elicited muscle damage in KO but not littermate mice (elevated serum CK; Supplemental Figure 4B). However, forelimb grip strength, a crude measure for detecting muscle weakness, was not different in $\mathrm{KO}$ mice and littermates (Figure 3D). Male MCK-Cre/Fktn (E17) KOs had a tendency toward muscle hypertrophy (similar to MCK-Cre/ dystroglycan $\mathrm{KO}$; ref. 30), but body weights were not significantly different (Figure 3E). Therefore, dystroglycan glycosylation disruption at E17 in differentiating muscle initiates a disorder consistent with mild, progressive muscular dystrophy.

Skeletal muscle FKTN deficiency initiated at E8 in early myogenesis is sufficient to induce severe muscle weakness and reduced viability in mice, a phenotype similar to that of congenital muscular dystrophy patients. In contrast to the MCK-Cre/Fktn (E17) KO mouse line, KO mice with Fktn deletion initiated during skeletal muscle specification at E8 (myf5-Cre/Fktn) were severely affected. KO mice were significantly underweight in both body weight and isolated calf weight (gastrocnemius plus soleus) compared with littermates at all ages tested (Figure 4E, calf not shown). Many KOs required gruel feeding in the cage, as they were too small or weak to reach food pellets suspended overhead. Despite the gruel feeding, $11 \%$ of myf5-Cre/Fktn $\mathrm{KO}$ mice died before 20 weeks of age, and $78 \%$ of mice that we attempted to age past 20 weeks died or required euthanasia under our IACUC protocol (for inability to feed, hind limb paralysis, or respiratory distress) before 35 weeks of age (Supplemental Figure 1E). Therefore, Fktn deletion initiated with skeletal muscle development (E8), but not with muscle differentiation (E17), reduced mouse lifespan, indicating an important role for functionally glycosylated dystroglycan in muscle development, differentiation, or regeneration.

Analysis of iliopsoas muscle from 20-week-old mice revealed moderate to severe dystrophic features in myf5-Cre/Fktn (E8) KOs compared with littermates (Figure 4, A and B). Dystrophy was particularly severe in 4 of 9 mice evaluated histologically; muscle fibers were sparse and interspersed with adipocytes and endomysial fibrosis (Figure 4A). The $\alpha$ DG functional glycan was virtually negative in muscle fibers of these severe cases, as the only staining present was restricted to peripheral nerves and nerve twigs (Figure 4A, e.g., asterisk). Surprisingly, a population of myf5-Cre/Fktn (E8) KOs exhibited patchy $\alpha$ DG staining (Figure 4A). Dystrophic features were observed in all of these mice, but they retained more muscle fibers and muscle mass than severely affected mice at 20 weeks of age.

To test whether other muscle groups in myf5-Cre/Fktn (E8) KO mice are affected, we also evaluated quadriceps, tibialis anterior, calf, and diaphragm muscles from the same mice represented in Figure 4A (Supplemental Figure 5). The diaphragm and iliopsoas muscles exhibited the most prevalent $\mathrm{KO}$ histopathology, consistent with a greater involvement of proximal muscles in dystroglycanopathies. Hind limb muscles were clearly dystrophic, but less severely affected. Similar to the iliopsoas, patchy expression of glycosylated $\alpha \mathrm{DG}$ was found in some muscles (Supplemental Figure 5). As expected, reduced $\alpha D G$ glycosylation was roughly correlated with the severity of dystrophy. This spectrum of disease in myf5Cre/Fktn (E8) KOs suggests that either Fktn deletion is incomplete or compensatory mechanisms for $\alpha \mathrm{DG}$ glycosylation are at work in the moderately affected mice (see below).

As a group, the early muscle KOs showed other disease features. CK activity in serum was elevated at 4 and 8 weeks of age for myf5-
Cre/Fktn (E8) KOs compared with littermates, indicating the presence of significant muscle damage early in life (Figure 4C). As expected, the CK activity decreased at older ages because fewer muscle fibers remain to contribute to protein leakage into serum. In addition, forelimb grip strength was significantly weaker in $\mathrm{KO}$ mice at nearly all ages tested (Figure 4D), confirming the clinical relevance of this disease model.

Using a transgenic mouse line developed by our group (TgMCKFktn; Supplemental Figure 6), we reexpressed the Fktn cDNA under control of the MCK promoter to direct exogenous FKTN expression at E17 in muscle of myf5-Cre/Fktn (E8) KO mice. We tested mice at PD8 to assess exogenous Fktn expression and dystrophic pathology. While we did not obtain a transgene-expressing $\mathrm{KO}$ mouse $(\mathrm{Tg} / \mathrm{KO})$ in PD8 litters, we did find that FKTN protein and mRNA were highly elevated by PD8 in transgeneexpressing littermate control mice $(\mathrm{Tg} / \mathrm{LC})$, with no disruption of $\alpha$ DG glycosylation or muscle histology (Supplemental Figure 7, A-C). In contrast, myf5-Cre/Fktn (E8) KO muscle lacking the transgene showed nearly complete loss of glycosylated $\alpha \mathrm{DG}$ by P8, but no overt necrosis (Supplemental Figure 7, A and C). These data suggest that exogenous Fktn expression precedes the onset of dystrophic pathology in TgMCK-Fktn $\times$ myf5-Cre/Fktn KO mice, as disruption of $\alpha \mathrm{DG}$ glycosylation in the $\mathrm{E} 8 \mathrm{KO}$ mice is delayed by a complex sequence of events (Cre expression $\rightarrow$ Fktn gene excision $\rightarrow$ depletion of Fktn mRNA $\rightarrow$ depletion of FKTN protein $\rightarrow$ disruption of $\alpha$ DG processing $\rightarrow$ depletion of wild-type $\alpha$ DG $\rightarrow$ initiation of disease).

We also analyzed mice from the Fktn transgene-expressing myf5Cre/Fktn KO colony at 20 weeks. At this age, changes in $\alpha$ DG glycosylation, weakness, or muscle damage in transgene-expressing $\mathrm{KO}$ mice $(\mathrm{Tg} / \mathrm{KO})$ were not observed, while myf5-Cre/Fktn (E8) KO littermates exhibited disease (Supplemental Figure 8). Fktn replacement successfully prevented loss of $\alpha$ DG glycosylation and dystrophic disease in transgene-expressing $\mathrm{KO}$ mice. Consequently, the TgMCK-Fktn $\times$ myf5-Cre/Fktn KO colony is expected to be a model of Fktn gene therapy.

Muscle regeneration in dystroglycanopathy. Fktn excision initiated at E8 in our myf5-Cre/Fktn KO model disrupts $\alpha$ DG glycosylation in muscle development such that all muscle life-cycle stages should be affected (development, differentiation, fiber maintenance, and muscle regeneration). The milder phenotype of the MCK-Cre/ Fktn (E17) KO mice, in which the $\alpha$ DG defect is limited to mature muscle fibers, suggests that $\alpha \mathrm{DG}$ abnormalities in muscle development, differentiation, and/or regeneration are important for the development of more severe disease phenotypes. To directly address the effects of $\alpha D G$ glycosylation defects in muscle regeneration, we analyzed expression of the embryonic isoform of myosin heavy chain (eMHC), a marker of regenerating fibers, in 20-week-old mice from the myf5-cre (E8) and MCK-cre (E17) muscle KO colonies. Muscle fibers positive for $\mathrm{MHC}$ were present in $\mathrm{KO}$ mice from both colonies, as expected for dystrophic muscle (Supplemental Figure 9A). Notably, eMHC-positive fibers were smaller in myf5cre/Fktn (E8) KO than MCK-cre/Fktn (E17) KO mice, and the 4 severely affected mice in the myf5-cre/Fktn (E8) KO data set had much higher eMHC fiber density (number of eMHC fibers per total muscle section area; 4 highest data points obtained from severely affected mice; Supplemental Figure 9B).

Smaller eMHC-positive fibers, coupled with the higher number of fibers in myf5-Cre/Fktn (E8) KO mice with severe pathology, indicate that $\alpha$ DG glycosylation abnormality may cause regenerating 
A

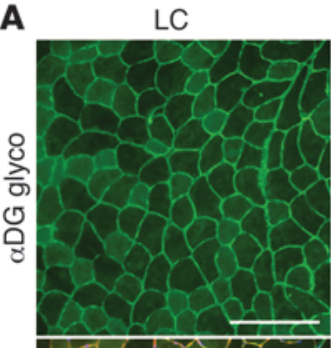

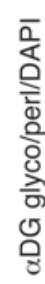
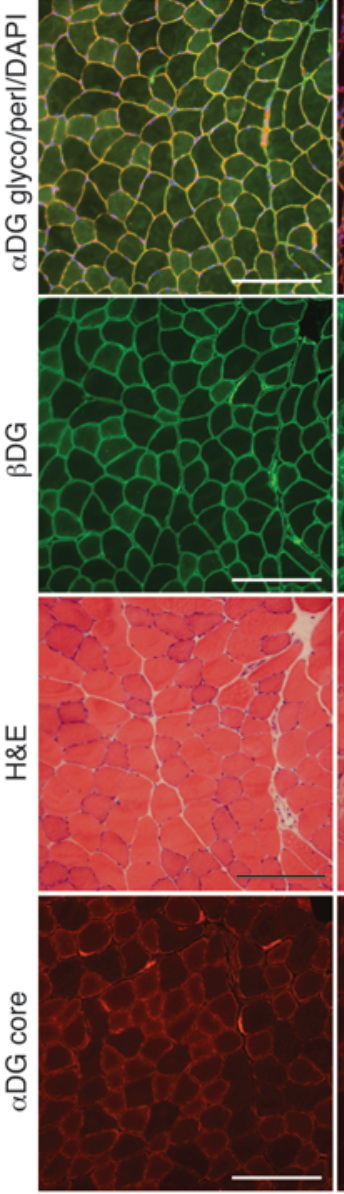

Myf5 KO
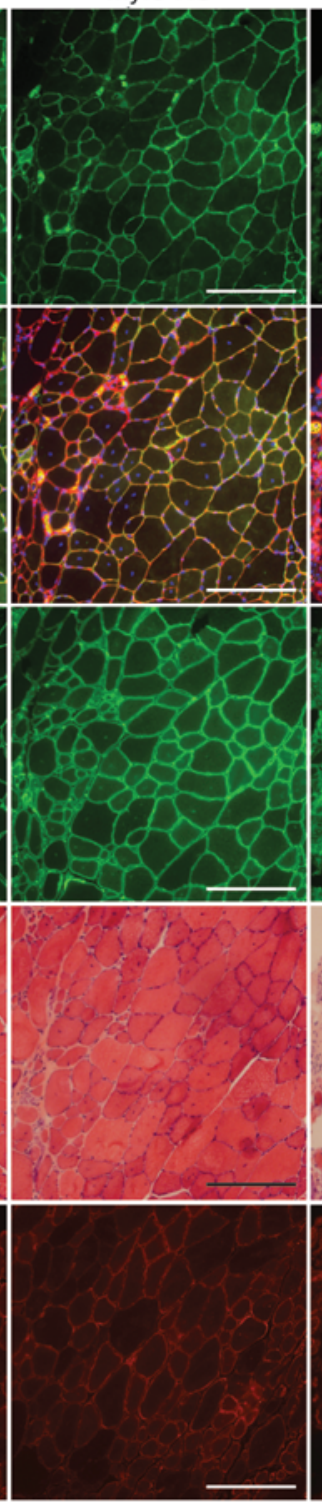

Myf5 KO
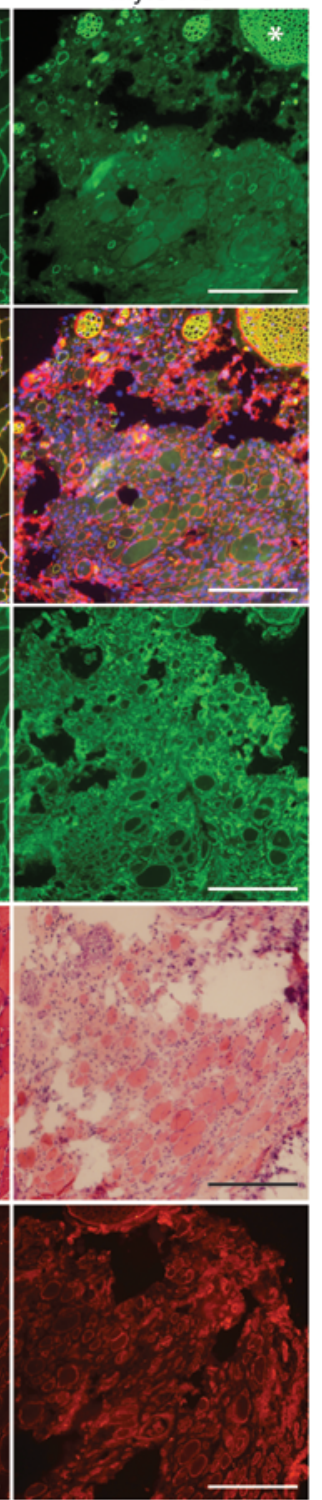

B

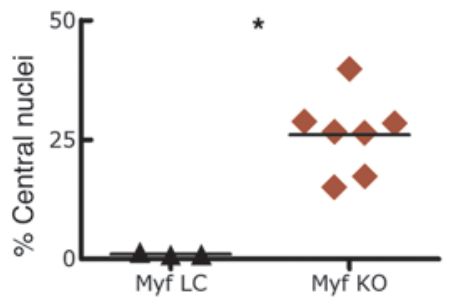

C

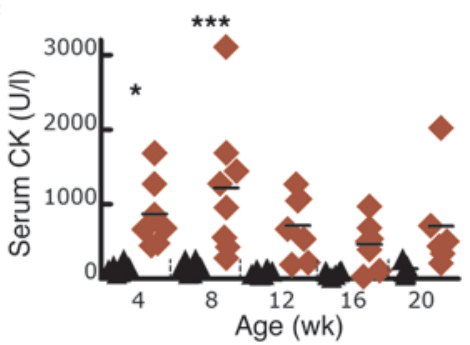

D

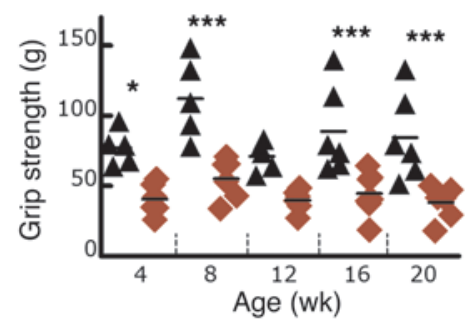

$\mathbf{E}$
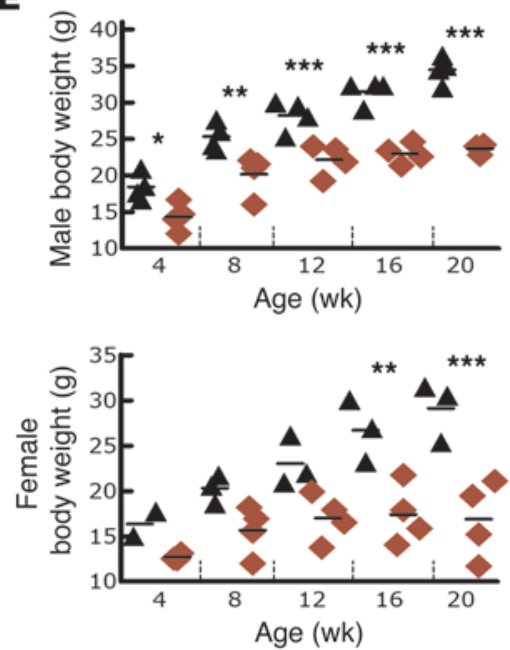

Figure 4

Mice with skeletal muscle Fktn deletion initiated at E8 show moderate to severe muscular dystrophy. (A) Histology of iliopsoas muscle from 20-week-old myf5-Cre LC and KO mice provides evidence of moderate to severe dystrophy. Patchy or absent staining with $\alpha \mathrm{DG}$ glyco-antibody while $\beta D G$ and $\alpha D G$ core protein are still present indicates abnormal $\alpha D G$ glycosylation. Original magnification, $\times 20$; scale bars: $100 \mu \mathrm{m}$. Note: Peripheral nerve twigs are still $\alpha D G$ glyco positive, confirming specificity of $F k t n$ KO in muscle (e.g., asterisk). (B) Iliopsoas fibers with central nucleation in individual mice at 20 weeks of age. ${ }^{*} P=0.017$; Mann Whitney test. (C) CK activity is elevated in KO mice at young ages. ${ }^{\star} P=0.01-0.05$, 4-week Myf LC versus KO; ${ }^{* *} P<0.001$, 8-week Myf LC versus KO; Bonferroni test. (D) Forelimb grip strength (average of 5 pulls) is plotted according to age ( ${ }^{*} P=0.01-0.05$, 4-week Myf LC versus KO; ${ }^{\star * \star} P<0.001$, 8-, 16-, and 20-week Myf LC versus KO pairs; Bonferroni test. (E) Body weights of male and female mice at various ages. ${ }^{*} P=0.01-0.05$, 4-week Myf LC versus KO M; ${ }^{\star *} P=0.001-0.01,8$-week Myf LC versus KO male and 16-week Myf LC versus KO female; ${ }^{* * \star} P<0.001,12-, 16-$, and 20-week Myf LC versus KO paired male and 20-week Myf LC versus KO female; Bonferonni test. Each data point represents 1 mouse; group means are shown. Black triangles, myf5-Cre LC; red diamonds, myf5-Cre Fktn-KO. Statistics were calculated for LC versus KO mice at each age. 
fibers to become "stalled" in an immature state. To determine whether these $\mathrm{KO}$ mice can mount a regenerative response in the context of advanced disease, we injected cardiotoxin (CTX; muscle toxin) or saline into calf muscles of 16-week-old myf5-Cre/Fktn (E8) $\mathrm{KO}$ and littermate mice. At 7 days after injection, we found CTX-induced muscle damage in both E8 KO mice and littermates and an increase in eMHC-positive regenerating fibers related to the size of the CTX necrotic region (Supplemental Figure 9, C and D). The distribution of eMHC-positive fiber areas was significantly smaller in myf5-Cre/Fktn (E8) KO mice than in littermates following synchronized CTX necrosis (Supplemental Figure 9E). Long-term recovery from CTX was also analyzed in myf5-Cre/Fktn (E8) KO mice. CTX-treated $\mathrm{KO}$ muscle was indistinguishable from saline-treated $\mathrm{KO}$ muscle 4 weeks after injection $(n=2$, data not shown); therefore, we could not determine whether myf5-Cre/Fktn $\mathrm{KO}$ muscle fully recovered to its dystrophic baseline 4 weeks after injection or if the CTX injection was off target. Overall, these data suggest that $\alpha D G$ glycosylation is not required for satellite cells to initiate a regenerative response; rather, it may be important for later stages of the regenerative process (fiber differentiation?). These results are consistent with reports of delayed fiber maturation in FCMD patients (31).

Variability between severe and moderate disease phenotypes. The apparent reemergence of $\alpha \mathrm{DG}$ glycosylation in some myf5-Cre/Fktn (E8) KO muscle fibers was unexpected, but correlated with reduced histopathology (see above). A modified immunofluorescence protocol with in situ denaturation and blocking of resident mouse immunoglobins was used with the $\alpha$ DG glyco-antibody in all experiments, as it was found to improve signal. When we compared signal detection in myf5-Cre/Fktn (E8) KO with or without the in situ denaturation, we found that denaturation increased the $\alpha$ DG membrane signal and background immunofluorescence. Therefore, it is possible that $\alpha \mathrm{DG}$ glycosylation in KO tissue may be overestimated (Supplemental Figure 10). However, because we did detect minimal amounts of glycosylated $\alpha$ DG biochemically (Figure 2), our data indicate that very little functional $\alpha D G$ is present in mice with reduced disease (Figure 2).

How is some $\alpha \mathrm{DG}$ glycosylation restored in myf5-Cre/Fktn (E8) $\mathrm{KO}$ muscle? One possible explanation for these results is that Cremediated excision of Fktn exon 2 is incomplete. If a small population of nuclei continued to produce wild-type FKTN, then $\alpha \mathrm{DG}$ glycosylation could be restored in some fibers. We prepared RNA from mouse calf muscles and used RT-PCR to detect FKTN transcripts. In 20-week-old mice, full-length Fktn and alternatively spliced variants were present in wild-type littermates, while only recombined transcripts ( $\Delta$ exon 2 ) were detected from the myf5Cre/Fktn (E8) KO mice (Supplemental Figure 11A), suggesting complete Cre recombination at all Fktn loci. We also assessed the number of Fktn and homolog Fkrp transcripts in muscle of 20-week-old KO and littermate mice. Fkrp levels were unchanged or slightly reduced in Fktn KO muscle (data not shown). Quantitative PCR (qPCR) methods were deemed unreliable for Fktn mRNA quantification because of low expression levels and the presence of other cell types (e.g., fibroblasts, adipocytes) in the dystrophic muscle compartment (data not shown).

As the absence of wild-type transcripts in the 20-week-old KO calf may not be representative of all muscle groups, we conducted further testing to assess Fktn recombination in other muscles. We analyzed older mice (27 weeks) to select for moderate phenotypes, as the most severely affected myf5-Cre/Fktn (E8) KOs die by this age. Under these conditions, wild-type transcript was faintly detected in several muscles, but was not consistently expressed in specific muscle groups across individual mice (Supplemental Figure 11B). While a portion of the unrecombined Fktn transcript was likely derived from peripheral nerve contamination of the muscle sample, these data suggest that a small population of muscle cells escape Cre recombination to support residual and mosaic $\alpha D G$ glycosylation. It seems unlikely that the residual unrecombined transcript accounts for all of the glycosylated $\alpha$ DG in moderately affected mice. Thus, we expect that additional molecular events contribute to the recovery of $\alpha \mathrm{DG}$ glycosylation in myf5-Cre/Fktn (E8) KO muscle, such as the variable expression of modifier genes.

\section{Discussion}

Dystroglycan roles in skeletal muscle. Our Fktn conditional $\mathrm{KO}$ mice provide, for the first time to our knowledge, a dystroglycanopathy model with specific disruption of dystroglycan glycosylation in muscle that enables recapitulation of both severe and mild disease phenotypes. We find that functional dystroglycan glycosylation is important in muscle development/differentiation and fiber maintenance, but that defects initiated at E8 (expected to affect muscle development/differentiation) appear necessary to induce more severe disease with prevalent weakness and wasting. The necessity of functional $\alpha \mathrm{DG}$ glycosylation in fiber maintenance is demonstrated in MCK-Cre/Fktn KO mice where Fktn deletion is initiated in muscle at E17. In these mice, Fktn excision is expected to occur late in the muscle differentiation process, likely ensuring that $\alpha \mathrm{DG}$ function is preserved until fiber maturation. Late onset ( $\sim 12$ weeks) of muscle damage (elevated serum CK) in the absence of detectable forelimb muscle weakness suggests that while DG processing by FKTN is imperative in skeletal muscle maintenance, the loss of its function in mature skeletal muscle results in only mild pathology and physical impairment that may only be manifested under conditions of muscle stress (i.e., exercise).

Our results with the Tam iKO mouse model confirm a role for FKTN processing of $\alpha$ DG in muscle maintenance. In these mice, all muscle development and differentiation occurred normally, as Fktn disruption was not initiated until Tam delivery at 6 weeks. In muscle, only fiber maintenance and regeneration after Tam delivery were affected. Notably, muscle damage (elevated serum CK) was detected in these mice just 8-10 weeks after treatment, even though mice were in a slower, mature growth phase, and thus rapid tissue expansion contributed less to the depletion of functional $\alpha D G$. While this mouse model is not muscle specific, the effects of dystroglycanopathy on brain are generally developmental $(6,20,22,32-36)$ and cardiac abnormalities usually of late onset (37). Therefore, the more rapid and sustained muscle damage in Tam iKO mice versus MCK-Cre/Fktn (E17) KO mice likely indicates an additive effect of $\alpha D G$ disruption during muscle regeneration and Fktn disruption in peripheral nerves.

Our finding that the regenerating fibers in myf5-Cre/Fktn (E8) $\mathrm{KO}$ mice were smaller in size than those in either MCK-Cre/Fktn (E17) KO mice or CTX-challenged littermates is intriguing (Supplemental Figure 9), but the physiological relevance of this shift in fiber size is currently unclear. The eMHC-positive fiber pool in dystrophic muscle has been shown to be of smaller fiber area than eMHC-negative fibers in either dystrophic or control muscle (38), but to our knowledge the size distribution of eMHC-expressing fibers has not been measured for control muscle in the context of induced regeneration. Notably, our data are consistent with the 
idea that FCMD patient muscle undergoes maturational arrest, with a failure to complete terminal differentiation (31), as an increase in eMHC-positive fibers and a smaller fiber area could be explained by a delay or deficit in muscle fiber maturation.

Interestingly, our data and those of Taniguchi et al. (31) suggest that the roles of the dystroglycan protein (complex) and $\alpha \mathrm{DG}$ glycosylation per se may be different. We previously reported that CTX induction of muscle regeneration is severely compromised in MORE-DG null mice, in which the dystroglycan gene (Dag1) is deleted at E5 (30), but is normal when Dag1 deletion is delayed (E17 MCK expression), indicating that dystroglycan expression in satellite cells is necessary for the regenerative response. In contrast, CTX-induced myf5-cre/Fktn (E8) KO mice, Largemyd/myd mice, and FCMD patients exhibit an increase in eMHC-expressing muscle fibers (Supplemental Figure 9 and ref. 31). Therefore, we propose that satellite cell regeneration may depend not specifically on $\alpha \mathrm{DG}$ glycosylation, but rather on the presence of dystroglycan protein and the DGC. Proper glycosylation may be important for correct muscle fiber differentiation during development or following regeneration, but not for the initial regenerative response. Our evidence that dystrophic muscle from Fktn-deficient (E8) mice remains capable of mounting a regenerative response at 16 weeks of age suggests that there may be a wide window for therapeutic intervention if gene- or protein-based therapies were to target new eMHC-positive fibers in late-stage dystrophic muscle for the normalization of $\alpha D G$ glycosylation.

Timing of $\alpha D G$ disruption and onset of pathology. Cre recombinase activation by myf5 at E8 and subsequent Fktn excision take place in myotome-specified cells in our myf5-Cre/Fktn (E8) KO mice. However, because the fate of endogenous FKTN protein expression could not be determined, we were unable to conclude the precise timing of FKTN protein depletion. There will be a delay between Fktn excision and subsequent depletion of properly processed $\alpha D G$ protein. Therefore, dystroglycanopathy processes are likely not initiated until late in muscle development or during the differentiation process. While we did not determine the precise developmental timing of $\alpha$ DG disruption in our myf5-Cre/Fktn E8 $\mathrm{KO}$ mice, our data indicate that the time course of virtually complete $\alpha D G$ protein turnover is less than 18 days ( $\alpha$ DG glyco disruption in PD8 myf5-Cre [E8] KO mice [Supplemental Figure 7]; and loss of $\alpha$ DG glyco 2.5 weeks after Tam dosing in Tam iKO mice [Supplemental Figure 2]). Furthermore, our data imply that the time to onset of overt dystrophic pathology is 19-39 days in myf5 E8 KO mice (pathology not apparent at PD8, but present by 4 weeks of age) and 28-42 days in the Tam iKO mice (Supplemental Figure 2C, overt dystrophy absent at 4 weeks but present at 6 weeks after treatment), indicating that there is considerable overlap in the onset of dystrophic processes in these different models. An earlier onset of $\alpha$ DG dysfunction alone cannot explain the increased disease severity in myf5-Cre/Fktn $\mathrm{KO}$ mice, as neither Tam iKO nor MCK-Cre/Fktn (E17) KO mice develop measurable weakness or wasting, even when aged to 20 weeks. These data support the notion that loss of functional $\alpha \mathrm{DG}$ in muscle development or differentiation processes (that are unaffected in the milder Tam iKO and MCK-Cre/Fktn [E17] KO mice) is necessary to achieve a severe dystroglycanopathy phenotype.

The classical model of membrane-related muscular dystrophy disease mechanisms suggests that contraction-induced mechanical damage to the plasma membrane or its ion channels precede cycles of degeneration and regeneration, leading to a depletion of muscle regenerative capacity and cumulative muscle dysfunction
(39-45). In fact, such an account is consistent with our finding in MCK-Cre/Fktn (E17) KO and Tam iKO mice, as the 8- to 12-week time frame from Fktn deletion to elevated serum CK allows for depletion of FKTN protein, loss of $\alpha$ DG functional glycosylation, depletion of wild-type $\alpha \mathrm{DG}$, and subsequent accumulation of contraction-induced muscle damage. However, this model is insufficient to describe severe congenital muscular dystrophies, as the early disease onset and severity allow less time for such cumulative damage processes. The congenital model of laminin deficiency shows less evidence of membrane damage than dystrophin-related muscular dystrophy (46), and dystroglycanopathies exhibit abnormalities in muscle fiber maturation (31) and muscle organization $(36,47-49)$, indicating that other mechanisms likely contribute to the severity of congenital diseases.

Relevance to clinical phenotypes and patient FKTN mutations. In general, pathogenic mutations in dystroglycanopathy genes must interrupt end-point $\alpha \mathrm{DG}$ functional glycosylation. In myf5-Cre/ Fktn (E8) KO mice, the proportion of glycosylation disruption was clearly linked with disease phenotype. While we anticipate that our immunofluorescence detection of the glyco-epitope may overestimate residual glycosylated $\alpha D G$, mice with more glyco-positive fibers clearly had a moderate, rather than severe, dystrophic phenotype in muscle. The source of this variability in myf5-Cre/Fktn (E8) KO mice may be, at least in part, due to a technicality of the promoter system. Roughly $10 \%$ of satellite cells are reported to be myf5 negative (50), and although myf5 KO mice are developmentally delayed, they are able to generate skeletal muscle through a myf5-null, myoD-dependent precursor cell pool $(51,52)$. Therefore, in some mice a small population of myofibers may escape Cre recombination, leading to more moderate disease phenotypes. These data indicate that mechanisms directing expansion of this presumably myf5-negative, myoD-positive pool of unrecombined precursor cells may result in variable skeletal muscle developmental pathways in our mice.

Regardless of the source, the correlation between the magnitude of residual glycosylation and disease phenotype in Fktn-KO mice is consistent with both previous mouse studies and patient reports. For example, $0 \%$ dystroglycan laminin binding is lethal in the mouse (e.g., Dag1, Pomt1, Fktn KOs; refs. 16, 18, 23), approximately $5 \%$ laminin binding activity is associated with severe disease, and dystroglycan laminin binding activity of 50\% (or greater) is not pathogenic (21). Similarly, dystroglycanopathy patients with elevated residual $\alpha$ DG glycosylation trend to have the milder limb-girdle phenotype, whereas patients with more severe glycosylation defects develop congenital muscular dystrophies with earlier onset and greater functional impairment $(53,54)$, although there are exceptions (55). With respect to FKTN, a patient homozygous for an early nonsense mutation died at 4.5 months, and patients with compound heterozygosity with the Japanese founder mutation and nonsense, frameshift, or exon-skipping mutations are clinically more severely affected than those homozygous for the founder mutation, indicating that disease severity is dependent on the nature of the gene mutation $(9,56,57)$. While we do not yet know the specific activity of FKTN in the $\alpha$ DG processing pathway, we presume that the degree of $\alpha \mathrm{DG}$ dysfunction and the disruption of FKTN protein activity will also be positively correlated to the FKTN gene mutation, as has been shown for POMT1 and POMGnT1 enzyme activity in patient cells $(58,59)$.

Conditional KO technology allowed us to excise Fktn at different times in a muscle-specific or whole-animal manner (E8, E17, 
6 weeks) to gain new knowledge of dystroglycanopathies, but such selective excision of Fktn at specific times and locations is not a natural phenomenon. Our results indicate that $\alpha \mathrm{DG}$ dysfunction during development/differentiation is necessary for the generation of severe phenotypes in mice; therefore, the specific correlation with patients is less obvious, because patient FKTN mutations are present in the germline. A link between phenotypic severity and FKTN dysfunction in muscle development/differentiation could be accounted for by two general mechanisms.

First, a FKTN patient mutation could have an identical effect on abnormal $\alpha$ DG species in developing and mature muscle, but other factors confer increased severity during developmental/differentiation processes. For example, FKTN expression could be upregulated during compared with after muscle development such that the $\alpha \mathrm{DG}$ functional binding glycan is more dependent on FKTN modifications in the E8-E17 period, resulting in more severe disease. Such a developmental upregulation has been described for Fktn in mouse brain, but expression levels in embryonic muscle are unknown (60-62). Skeletal muscle may also be more sensitive to dystroglycan glycosylation abnormalities initiated in utero and early postnatal life because of the rapid tissue growth associated with development. In addition, modifier genes or proteins may be developmentally regulated so that the impact of abnormal $\alpha D G$ function is dependent on their coexpression. As these scenarios affect all FKTN patients, the specific output of patient phenotype is thus dependent on the percentage of residual functional dystroglycan-matrix binding as determined by the patient mutation, with greater residual binding conferring milder phenotypes.

Second, specific FKTN patient mutations could confer selectivity for enhanced dystroglycan glycosylation abnormalities during muscle development/differentiation versus after muscle development. For example, specific FKTN mutations could alter embryonic splicing or specific interactions with developmentally regulated molecules that determine FKTN RNA or protein stability or localization. A retrotransposon insertion into the $3^{\prime}$ untranslated region of FKTN, the Japanese founder mutation, has now been shown to cause a splicing defect resulting in truncation of the $\mathrm{C}$ terminus and addition of exogenous sequence that results in mislocalization to the ER (63). Penetrance of the FKTN splicing defect was variable in patient lymphoblasts, and multiple alternatively spliced FKTN mRNA human variants have been reported previously $(63,64)$. Therefore, the milder nature and variability of phenotype for patients homozygous for the founder mutation support the idea that differences in regulation of its pathogenic splicing are a possible source of patient variability that could be developmentally regulated.

Dystroglycanopathy disease models. Efforts to produce models of dystroglycanopathy syndromes have been made by numerous laboratories. As dystroglycan and the putative glycosylation machinery are largely conserved, invertebrate, vertebrate, and mammalian models have been generated. Lower organisms have proven efficient in corroborating the importance of dystroglycan and its processing enzymes $(36,47-49)$, but they are less suited for detailed analyses of downstream muscle pathogenesis. The essential role of dystroglycan in embryonic development (formation of Reichert's membrane) makes the use of traditional mouse KO technology difficult. Our data confirm a previous report of embryonic lethality in germline Fktn-KO mice (17) and are consistent with E6.5-E9.5 lethality reported for other primary and secondary dystroglycanopathy genes, Dag1, Pomt1, Pomt2, and Fkrp (16, 18, 20, 65). Embryonic lethality is expected for Large, but has not been reported.
To avoid lethality of germline $\mathrm{KO}$, chimeric mice and patient mutation knock-in mice have been studied, with mixed results. Chimeric mice with a high proportion of Fktn-null cells develop early, severe muscle disease, but dystrophy diminishes with time as wild-type satellite cell precursors repopulate the regenerating muscle (23). Moreover, chimeric mice are subject to increased inter-individual variability and repeated generation of chimeric mice for new studies is technically demanding and costly. FKRP patient mutation, humanized FKTN retrotransposon insertion, and Pomgnt1 genetrap mouse models exhibit only mild or nonexistent skeletal muscle histopathology $(19,21,66)$, indicating that gene disruption via patient mutation is subclinical (FKRP, FKTN) or that modifications are less critical or are compensated in muscle (Pomgnt1). FKRP knock-in mice with concomitant depletion of transcript mRNA successfully recapitulate secondary dystroglycanopathies, suggesting that point mutations alone do not disrupt protein activity sufficient enough to result in myopathies (20, 22). Unfortunately, these mice have significant perinatal lethality, precluding many mechanistic and therapeutic evaluations.

The most widely used dystroglycanopathy mouse models to date are the myodystrophy mouse (Large myd/myd $^{\text {) with spontaneous }}$ intragenic deletion of Large (11) and the conditional KO model of floxed dystroglycan $(30,35,67)$. Large $e^{m y d} / m y d$ mice have defective dystroglycan glycosylation, causing muscular dystrophy with brain and eye phenotypes, with specific abnormalities in muscle cytoarchitecture, neuromuscular junctions, and fiber maturation $(31,33,37,68)$. However, because the whole animal is affected in Large myd/myd $^{\text {mice, }}$ it is difficult to discriminate among myogenic, neurogenic, and vascular pathomechanisms. We have previously reported a conditional model of primary dystroglycanopathy using Cre/LoxP excision of the dystroglycan gene $(30,67)$. The floxed dystroglycan model has a loss of dystroglycan and the DGC (30) and therefore is not favorable for investigating cellular mechanisms of common secondary dystroglycanopathy, where glycosylation but not protein expression is abnormal. Hu et al. generated the first secondary dystroglycanopathy conditional mouse model using Cre-LoxP technology to remove Pomt2 in the brain (65). These mice recapitulate dystroglycanopathy brain defects, but gene disruption in muscle was not performed. Therefore, the conditional Fktn-KO mouse generated in this study, like the conditional Pomt2 mouse, offers substantial advantages over previous models, as it allows for the generation of tissue- and timingspecific defects in dystroglycan glycosylation, avoids embryonic lethality, and has a phenotype that resembles patient pathology. Furthermore, the detailed analysis of disease progression in skeletal muscle outlined in this study will be helpful for determining milestones in future therapeutic animal studies.

Insights into FKTN activity. It is well established that $\alpha \mathrm{DG}$ is modified by $O$-linked glycosylation $(1,69-71)$ and that $O$-mannosespecific glycosylation is responsible for $\alpha \mathrm{DG}$ binding to basement membrane proteins $(4,72-76)$. FKTN is implicated in the $\alpha D G$ processing pathway because its mutations disrupt $\alpha$ DG glycosylation $(37,77)$; however, neither the complete $\alpha$ DG $O$-mannose structure nor the site of FKTN's activity is known. Our data, in agreement with a recent study, demonstrate that the abnormal glycosylation caused by Fktn deficiency is associated with a glycan truncation near the $\alpha \mathrm{DG} O$-mannose phosphate bond $(15,27)$. We find that Fktn-deficient $\alpha \mathrm{DG}$ is present in two populations, one with a free phosphate group attached to the $O$-mannose and one with a small glycan modification added to the phosphate (protein- 
O-mannose-P-X). While LicD domain-containing proteins have been shown to have phosphoryl transfer activity (78), we incorrectly anticipated that FKTN might itself be responsible for the $\alpha D G$ glycan phosphate group. However, our data confirm that the $\alpha \mathrm{DG} O$-mannose phosphate does not require FKTN (Figure 2C and Supplemental Figure 2); rather, FKTN activity is linked to modifications immediately downstream of the mannose phosphate residue. Notably, FKTN was previously reported to bind to the $\alpha$ DG processing enzyme POMGnT1, which adds $N$-acetylglucosamine to $\alpha \mathrm{DG} O$-mannose $(8,69,79)$, providing further evidence that FKTN activity is relevant to modifications near the $\alpha \mathrm{DG} O$-mannose sugar.

$\alpha \mathrm{DG}$ from Largemyd/myd mice has also been shown to be deficient at the $\alpha$ DG $O$-mannose phosphate; however, only the free phosphate $\alpha D G$ population was reported (15). In preparation for experiments presented here, we also analyzed Large $e^{\text {myd/myd }}$ muscle lysates on PHOS-beads. In contrast to the previous report, we detected two $\alpha$ DG populations from Large $e^{m y d / m y d}$ mice (data not shown), just as we found with our MCK-Cre/Fktn and myf5-Cre/Fktn KOs (Figure 2). The source of the discrepancy is not immediately clear. However, PHOS-bead voids require buffer exchange before SDSPAGE, which can deplete $30 \%-50 \%$ of sample proteins, so it is possible that, with a smaller starting sample, the $\alpha$ DG PHOS-bead void fraction was subthreshold for detection. Ultimately, we found that Fktn and Large deficiency produce similar $\alpha$ DG subspecies, suggesting that their activities converge in $\alpha \mathrm{DG}$ processing. The precise nature of the PHOS-bead void $\alpha \mathrm{DG}$ is not clear. However, we speculate that the free $\alpha \mathrm{DG} O$-mannose phosphate is the specific site of disruption in Fktn- (and Large-) deficient muscle and in the absence of its usual processing, other nonspecific modifications to the $\alpha \mathrm{DG}$ phosphate occur to generate the PHOS-bead void fraction. Further identification of the $\alpha \mathrm{DG} O$-mannose glycan is required to reconcile these questions.

\section{Methods}

Antibodies. Custom rabbit antiserum (rabbit 257) was generated against the human FKTN C terminus (KLH-CAANGIWPISEWDEVIQLY) using standard protocols. Affinity-purified Rbt257 detects recombinant FKTN protein by Western blot but lacks the sensitivity to detect endogenous protein; Rbt257 was not suitable for immunofluorescence detection. $\alpha$ DG glyco (IIH6), $\alpha$ DG core (goat 20adg), and $\beta$ DG (7D11) were described previously $(1,4,80)$. Perlecan (heparin sulfate proteoglycan) and laminin primary antibodies and secondary antibodies conjugated to Cy3, Alexa Fluor 488, HRP, IR dye 680/700 nm, and IR dye $800 \mathrm{~nm}$ were purchased (Millipore, Sigma-Aldrich, Jackson ImmunoResearch Laboratories Inc., Invitrogen, Chemicon, Pierce, Rockland).

Mice. Floxed Fktn mice for conditional deletion of Fktn exon 2 were generated by homologous recombination in mouse 129/SvE ES cells by the University of Iowa Gene Targeting Core Facility. Germline deletion of the neomycin selection cassette or the entire floxed exon 2 was achieved by mouse breeding. Conditional KO mice $\mathrm{Tg}^{\mathrm{Cre}-\mathrm{Esr} 1} ; \mathrm{Fktn}^{\mathrm{fl} /-}(\mathrm{Tam} \mathrm{iKO}), \mathrm{myf5}^{\mathrm{Cre} /+} ; \mathrm{Fktn}^{\mathrm{fl} / \mathrm{fl}}$ (myf5-Cre/Fktn KO), and MCK-Cre/Fktn mice were created by breeding to inducible or muscle-specific Cre recombinase mice. MCK-Cre mice were a gift from Ronald Kahn (Harvard Medical School, Boston, Massachusetts, USA) and myf5-Cre mice were a gift from Michael Rudnicki (Ottawa Hospital Research Institute, Ottawa, Ontario, Canada). For details, see Supplemental Methods and Supplemental Tables 1 and 2.

$R T$-PCR. Calf (gastrocnemius plus soleus) muscles from individual 20-week-old mice were flash frozen and crushed under liquid nitrogen. RNA was extracted from muscle using TRIzol according to the manu- facturer's instructions (Invitrogen). RNA concentration and quality were measured (Nanodrop). One microgram of RNA per sample was treated with DNase, reverse transcribed using a mixture of oligo dT and random hexamer primers (Superscript II, Invitrogen), and treated with RNase H to remove template RNA. FKTN exon 1-4 was amplified by PCR (Supplemental Table 3).

Physiological measurements. Body weight, forelimb grip strength, and serum CK measurements were taken at 2- or 4-week intervals from 4 to 20 weeks of age. A mouse grip strength meter (Columbus Instruments) was mounted horizontally, with a T-bar connected to the force transducer. Mice were allowed to grip the bar with their front paws and then were gently pulled away until the bar was released. The maximum gram force was recorded per pull, and 5 pulls were averaged per mouse for each time point.

Blood was collected from the ventral tail artery of individual mice and centrifuged to separate serum. Serum $(3 \mu \mathrm{l})$ was diluted 1:10 in ultrapure water and loaded onto a microplate in triplicate. Creatine kinase reagent (StanBio 0910-206) was added according to the manufacturer's protocol and assayed for activity using a microplate reader (Molecular Devices).

Lectin purification, PHOS-bead analysis, and laminin overlay. WGA-enriched protein samples were prepared from various tissues (hind limb skeletal muscle, brain, pooled peripheral nerves, kidney, liver, lung, thymus, and testes) or tissue cryosections (heart). Tissues were ground in solubilization buffer (150 mM NaCl, $50 \mathrm{mM}$ Tris, protease inhibitors [PIs], $\mathrm{pH}$ 7.4) with $1 \%$ Triton X-100. Solubilized supernatants were incubated with WGA agarose (Vector Laboratories). Void fractions were collected, beads were washed (solubilization buffer plus $0.1 \%$ Triton X-100), and protein was eluted with $0.3 \mathrm{M} \mathrm{N}$-acetylglucosamine.

For PHOS-bead analysis, WGA elution samples ( 5-8 times the amount of sample used for WGA SDS-PAGE analysis) were desalted by two 10-fold dilutions in water (Amicon Ultra spin columns, Millipore). Concentrated samples were mixed $1: 1$ with $2 \times$ buffer $(500 \mathrm{mM}$ acetic acid, $60 \%$ acetonitrile) and applied to PHOS-beads (Sigma-Aldrich) pre-equilibrated in $1 \times$ buffer $(250 \mathrm{mM}$ acetic acid, $30 \%$ acetonitrile). Samples were rotated for 30 minutes at room temperature and centrifuged at $8,200 \mathrm{~g}$, and void fractions were collected. PHOS-beads were washed twice with $1 \times$ buffer and once with $\mathrm{ddH}_{2} \mathrm{O}$. Proteins were eluted from the PHOS-beads with phosphate buffer (200 mM sodium phosphate monobasic, $\mathrm{pH}$ 8.4) and mixed with $5 \times$ Laemmli sample buffer $(5 \times$ LSB) for SDS-PAGE. PHOS-bead voids were buffer exchanged (same as desalting step) and concentrated, diluted 1:1 with phosphate buffer, and mixed with $5 \times$ LSB for SDS-PAGE. All protein samples were separated by $3 \%-15 \%$ SDS-PAGE and transferred to PVDF for Western blotting using standard protocols (81).

Laminin overlays have been described previously (37). Natural mouse laminin (Invitrogen) at $10 \mu \mathrm{g} / \mathrm{ml}$ was used for the overlay and was detected by rabbit polyclonal anti-laminin antibody (Sigma-Aldrich).

Histology, microscopy, and central nucleation. Seven-micrometer tissue cryosections were processed for hematoxylin and eosin (H\&E) staining according to standard protocols. For secondary immunofluorescence, tissues were blocked with 5\% donkey serum in PBS, incubated in primary antibody overnight, washed, incubated in Cy3 (Jackson ImmunoResearch Laboratories Inc.) or Alexa Fluor 488-conjugated (Molecular Probes, Invitrogen) secondary antibodies, washed, and coverslipped. With the exception of $\alpha$ DG glycosylation staining, tissue cryosections were processed for immunofluorescence as described previously (81). For $\alpha$ DG glyco-antibody IIH6, tissues were fixed in 2\% PFA, washed, and denatured by incubations with $100 \mathrm{mM}$ glycine at room temperature and $0.05 \%$ SDS at $50^{\circ} \mathrm{C}$ for 10 and 30 minutes, respectively (82). After washing, tissues were blocked with $5 \%$ donkey serum, then incubated with $100 \mu \mathrm{g} / \mathrm{ml} \mathrm{F}(\mathrm{ab})$ fragment from mouse IgM $\mu$ chain (Jackson ImmunoResearch Laborato- 
ries Inc.). Tissues were washed and incubated overnight with primary antibody in blocking solution; subsequent steps were performed as previously described (81). All solutions were made in PBS (Invitrogen).

Statistics. Data are plotted as scatter plots with group mean or box and whiskers plots (minimum to maximum). Statistical significance was calculated using the Mann-Whitney 2-tailed unpaired $t$-test when comparing only 2 groups. For data sets with more than 2 groups, a D'Agostino and Pearson normality test was performed. When 1 or fewer groups failed the normality test (or where normality could not be determined due to sample size), 1-way ANOVA was used with a Tukey post-test (when comparing all samples) or a Bonferroni's multiple comparison post-test (when detecting differences within age groups; e.g., grip strength). When 2 or more groups in the data set failed the normality test, a Kruskal-Wallis test was performed with Dunn's post-test on selected pairs (e.g., Figure 1C). All statistical analysis was calculated in GraphPad Prism; a $P$ value of less than 0.05 was considered significant. Biochemical data reflect a minimum of three experiments; central nucleation (number of fibers with central nuclei/total number fibers) was quantified from 3 representative microscope fields per mouse using ImagePro Express (Media Cybernetics) and plotted as the average percent central nuclei per mouse.

Study approval. All mouse generation, breeding, and experiments were approved by the University of Iowa and the University of Georgia Institutional Animal Care and Use Committees.

\section{Acknowledgments}

Baoli Yang and Kazuhiro Kobuke provided assistance in construct design. Rolf Turk, Tobias Willer, Takako Moriguchi, Steve Moore, Keith Garringer, Daniel Beltran, Sally Prouty, Rob Crawford, David Venzke, Erik Rader, and Mary Anderson gave technical assistance and/or helpful comments on this project. This work was funded, in part, by the Muscular Dystrophy Association with Research and Development grants (to K.P. Campbell, AMB \#114819, JDL \# 186940), the NIH Senator Paul D. Wellstone Muscular Dystrophy Cooperative Research Center (to K.P. Campbell), the Alberta Heritage Foundation for Medical Research (to A.M. Beedle), and the University of Iowa Peter A. Getting Award (to A.M. Beedle). K.P. Campbell is an investigator of the Howard Hughes Medical Institute.

Received for publication January 30, 2012, and accepted in revised form June 21, 2012.

Address correspondence to: Kevin P. Campbell, Howard Hughes Medical Institute, Department of Molecular Physiology and Biophysics, Carver College of Medicine, Director, Senator Paul D. Wellstone MDCRC, University of Iowa, 4269B CBRB, 285 Newton Road, Iowa City, Iowa 52242, USA. Phone: 319.335.7867; Fax: 319.335.6957; E-mail: kevin-campbell@uiowa.edu.
1. Ervasti JM, Campbell KP. Membrane organization of the dystrophin-glycoprotein complex. Cell. 1991;66(6):1121-1131.

2. Ervasti JM. Dystrophin, its interactions with other proteins, and implications for muscular dystrophy. Biochim Biophys Acta. 2007;1772(2):108-117.

3. Guglieri M, Magri F, Comi GP. Molecular etiopathogenesis of limb girdle muscular and congenital muscular dystrophies: boundaries and contiguities. Clin Chim Acta. 2005;361(1-2):54-79.

4. Ibraghimov-Beskrovnaya O, Ervasti JM, Leveille CJ, Slaughter CA, Sernett SW, Campbell KP. Primary structure of dystrophin-associated glycoproteins linking dystrophin to the extracellular matrix. Nature. 1992;355(6362):696-702.

5. Barresi R, Campbell KP. Dystroglycan: from biosynthesis to pathogenesis of human disease. J Cell Sci. 2006;119(pt 2):199-207.

6. Beltran-Valero de Bernabe D, et al. Mutations in the $\mathrm{O}$-mannosyltransferase gene POMT1 give rise to the severe neuronal migration disorder Walker-Warburg syndrome. Am J Hum Genet. 2002;71(5):1033-1043.

7. Willer T, Amselgruber W, Deutzmann R, Strahl S. Characterization of POMT2, a novel member of the PMT protein O-mannosyltransferase family specifically localized to the acrosome of mammalian spermatids. Glycobiology. 2002;12(11):771-783.

8. Yoshida A, et al. Muscular dystrophy and neuronal migration disorder caused by mutations in a glycosyltransferase, POMGnT1. Dev Cell. 2001;1(5):717-724.

9. Kobayashi K, et al. An ancient retrotransposal insertion causes Fukuyama-type congenital muscular dystrophy. Nature. 1998;394(6691):388-392.

10. Brockington $M$, et al. Mutations in the fukutin-related protein gene (FKRP) cause a form of congenital muscular dystrophy with secondary laminin alpha2 deficiency and abnormal glycosylation of alpha-dystroglycan. Am J Hum Genet. 2001;69(6):1198-1209.

11. Grewal PK, Holzfeind PJ, Bittner RE, Hewitt JE. Mutant glycosyltransferase and altered glycosylation of alpha-dystroglycan in the myodystrophy mouse. Nat Genet. 2001;28(2):151-154.

12. Manya H, et al. Demonstration of mammalian protein O-mannosyltransferase activity: coexpression of POMT1 and POMT2 required for enzymatic activity. Proc Natl Acad Sci U S A. 2004;101(2):500-505.

13. Zhang W, et al. Enzymatic diagnostic test for Mus-
cle-Eye-Brain type congenital muscular dystrophy using commercially available reagents. Clin Biochem. 2003;36(5):339-344.

14. Inamori K, Yoshida-Moriguchi T, Hara Y, Anderson ME, Yu L, Campbell KP. Dystroglycan function requires xylosyl- and glucuronyltransferase activities of LARGE. Science. 2012;335(6064):93-96.

15. Yoshida-Moriguchi T, et al. O-mannosyl phosphorylation of alpha-dystroglycan is required for laminin binding. Science. 2010;327(5961):88-92.

16. Williamson RA, et al. Dystroglycan is essential for early embryonic development: disruption of Reichert's membrane in Dag1-null mice. Hum Mol Genet. 1997;6(6):831-841.

17. Kurahashi $\mathrm{H}$, et al. Basement membrane fragility underlies embryonic lethality in fukutin-null mice. Neurobiol Dis. 2005;19(1-2):208-217.

18. Willer T, et al. Targeted disruption of the WalkerWarburg syndrome gene Pomt 1 in mouse results in embryonic lethality. Proc Natl Acad Sci U S A. 2004;101(39):14126-14131.

19. Liu J, et al. A genetic model for muscle-eye-brain disease in mice lacking protein $\mathrm{O}$-mannose 1,2-N-acetylglucosaminyltransferase (POMGnT1). Mech Dev. 2006;123(3):228-240.

20. Chan YM, et al. Fukutin-related protein is essential for mouse muscle, brain and eye development and mutation recapitulates the wide clinical spectrums of dystroglycanopathies. Hum Mol Genet. 2010;19(20):3995-4006.

21. Kanagawa M, et al. Residual laminin-binding activity and enhanced dystroglycan glycosylation by LARGE in novel model mice to dystroglycanopathy. Hum Mol Genet. 2009;18(4):621-631.

22. Ackroyd MR, et al. Reduced expression of fukutin related protein in mice results in a model for fukutin related protein associated muscular dystrophies. Brain. 2009;132(pt 2):439-451.

23. Takeda S, et al. Fukutin is required for maintenance of muscle integrity, cortical histiogenesis and normal eye development. Hum Mol Genet. 2003; 12(12):1449-1459.

24. Sauer B. Inducible gene targeting in mice using the Cre/lox system. Methods. 1998;14(4):381-392.

25. Sadowski PD. The Flp recombinase of the 2 -microns plasmid of Saccharomyces cerevisiae. Prog Nucleic Acid Res Mol Biol. 1995;51:53-91.
26. Ibraghimov-Beskrovnaya $\mathrm{O}$, et al. Human dystroglycan: skeletal muscle cDNA, genomic structure, origin of tissue specific isoforms and chromosomal localization. Hum Mol Genet. 1993;2(10):1651-1657.

27. Kuga A, et al. Absence of post-phosphoryl modification in dystroglycanopathy mouse models and wild-type tissues expressing non-laminin binding form of alpha-dystroglycan. J Biol Chem. 2012; 287(12):9560-9567.

28. Sabourin LA, Rudnicki MA. The molecular regulation of myogenesis. Clin Genet. 2000;57(1):16-25.

29. Trask RV, Billadello JJ. Tissue-specific distribution and developmental regulation of $\mathrm{M}$ and $\mathrm{B}$ creatine kinase mRNAs. Biochim Biophys Acta. 1990; 1049(2):182-188.

30. Cohn RD, et al. Disruption of DAG1 in differentiated skeletal muscle reveals a role for dystroglycan in muscle regeneration. Cell. 2002;110(5):639-648.

31. Taniguchi M, et al. Aberrant neuromuscular junctions and delayed terminal muscle fiber maturation in alpha-dystroglycanopathies. Hum Mol Genet. 2006;15(8):1279-1289.

32. Chiyonobu T, et al. Effects of fukutin deficiency in the developing mouse brain. Neuromuscul Disord. 2005;15(6):416-426.

33. Holzfeind PJ, et al. Skeletal, cardiac and tongue muscle pathology, defective retinal transmission, and neuronal migration defects in the Large(myd) mouse defines a natural model for glycosylationdeficient muscle - eye - brain disorders. Hum Mol Genet. 2002;11(21):2673-2687.

34. Moore SA, et al. Deletion of brain dystroglycan recapitulates aspects of congenital muscular dystrophy. Nature. 2002;418(6896):422-425.

35. Satz JS, et al. Distinct functions of glial and neuronal dystroglycan in the developing and adult mouse brain. J Neurosci. 2010;30(43):14560-14572.

36. Shcherbata HR, et al. Dissecting muscle and neuronal disorders in a Drosophila model of muscular dystrophy. EMBOJ. 2007;26(2):481-493.

37. Michele DE, et al. Post-translational disruption of dystroglycan-ligand interactions in congenital muscular dystrophies. Nature. 2002;418(6896):417-422.

38. Yuasa K, Nakamura A, Hijikata T, Takeda S. Dystrophin deficiency in canine X-linked muscular dystrophy in Japan (CXMDJ) alters myosin heavy chain expression profiles in the diaphragm more 
markedly than in the tibialis cranialis muscle. $B M C$ Musculoskelet Disord. 2008;9:1.

39. Han R, et al. Basal lamina strengthens cell membrane integrity via the laminin $\mathrm{G}$ domain-binding motif of alpha-dystroglycan. Proc Natl Acad Sci U S A. 2009;106(31):12573-12579.

40. Cohn RD, Campbell KP. Molecular basis of muscular dystrophies. Muscle Nerve. 2000;23(10):1456-1471.

41. Claflin DR, Brooks SV. Direct observation of failing fibers in muscles of dystrophic mice provides mechanistic insight into muscular dystrophy. $A m$ J Physiol Cell Physiol. 2008;294(2):C651-C658.

42. Dudley RW, Danialou G, Govindaraju K, Lands L, Eidelman DE, Petrof BJ. Sarcolemmal damage in dystrophin deficiency is modulated by synergistic interactions between mechanical and oxidative/nitrosative stresses. Am J Pathol. 2006;168(4):1276-1287.

43. Straub V, et al. Molecular pathogenesis of muscle degeneration in the delta-sarcoglycan-deficient hamster. Am J Pathol. 1998;153(5):1623-1630.

44. Petrof BJ, Shrager JB, Stedman HH, Kelly AM, Sweeney HL. Dystrophin protects the sarcolemma from stresses developed during muscle contraction. Proc Natl Acad Sci US A. 1993;90(8):3710-3714.

45. Allen DG, Zhang BT, Whitehead NP. Stretchinduced membrane damage in muscle: comparison of wild-type and mdx mice. Adv Exp Med Biol. 2010;682:297-313

46. Straub V, Rafael JA, Chamberlain JS, Campbell KP. Animal models for muscular dystrophy show different patterns of sarcolemmal disruption. $J$ Cell Biol. 1997;139(2):375-385.

47. Haines N, Seabrooke S, Stewart BA. Dystroglycan and protein $\mathrm{O}$-mannosyltransferases 1 and 2 are required to maintain integrity of Drosophila larval muscles. Mol Biol Cell. 2007;18(12):4721-4730.

48. Parsons MJ, Campos I, Hirst EM, Stemple DL. Removal of dystroglycan causes severe muscular dystrophy in zebrafish embryos. Development. 2002; 129(14):3505-3512.

49. Thornhill P, Bassett D, Lochmuller H, Bushby K, Straub V. Developmental defects in a zebrafish model for muscular dystrophies associated with the loss of fukutin-related protein (FKRP). Brain. 2008; 131(pt 6):1551-1561.

50. Kuang S, Kuroda K, Le Grand F, Rudnicki MA. Asymmetric self-renewal and commitment of satellite stem cells in muscle. Cell. 2007;129(5):999-1010.

51. Braun T, Rudnicki MA, Arnold HH, Jaenisch R Targeted inactivation of the muscle regulatory gene Myf-5 results in abnormal rib development and perinatal death. Cell. 1992;71(3):369-382.

52. Rudnicki MA, Schnegelsberg PN, Stead RH, Braun T, Arnold HH, Jaenisch R. MyoD or Myf-5 is required for the formation of skeletal muscle. Cell. 1993;75(7):1351-1359

53. Godfrey C, Foley AR, Clement E, Muntoni F. Dystroglycanopathies: coming into focus. Curr Opin Genet Dev. 2011;21(3):278-285.
54. Brown SC, et al. Abnormalities in alpha-dystroglycan expression in MDC1C and LGMD2I muscular dystrophies. Am J Pathol. 2004;164(2):727-737.

55. Jimenez-Mallebrera C, et al. A comparative study of alpha-dystroglycan glycosylation in dystroglycanopathies suggests that the hypoglycosylation of alphadystroglycan does not consistently correlate with clinical severity. Brain Pathol. 2009;19(4):596-611.

56 . Kondo-Iida E, et al. Novel mutations and genotype-phenotype relationships in 107 families with Fukuyama-type congenital muscular dystrophy (FCMD). Hum Mol Genet. 1999;8(12):2303-2309.

57. de Bernabe DB, et al. A homozygous nonsense mutation in the fukutin gene causes a WalkerWarburg syndrome phenotype. J Med Genet. 2003; 40(11):845-848.

58. Lommel $\mathrm{M}$, et al. Correlation of enzyme activity and clinical phenotype in POMT1-associated dystroglycanopathies. Neurology. 2010;74(2):157-164.

59. Vajsar J, et al. Carriers and patients with muscleeye-brain disease can be rapidly diagnosed by enzymatic analysis of fibroblasts and lymphoblasts. Neuromuscul Disord. 2006;16(2):132-136.

60. Saito Y, Mizuguchi M, Oka A, Takashima S. Fukutin protein is expressed in neurons of the normal developing human brain but is reduced in Fukuyama-type congenital muscular dystrophy brain. Ann Neurol. 2000;47(6):756-764.

61. Yamamoto T, et al. Fukutin expression in glial cells and neurons: implication in the brain lesions of Fukuyama congenital muscular dystrophy. Acta Neuropathol. 2002;104(3):217-224.

62. Horie M, Kobayashi K, Takeda S, Nakamura Y, Lyons GE, Toda T. Isolation and characterization of the mouse ortholog of the Fukuyama-type congenital muscular dystrophy gene. Genomics. 2002; 80(5):482-486.

63. Taniguchi-Ikeda M, et al. Pathogenic exontrapping by SVA retrotransposon and rescue in Fukuyama muscular dystrophy. Nature. 2011; 478(7367):127-131.

64. Kobayashi K, et al. Structural organization, complete genomic sequences and mutational analyses of the Fukuyama-type congenital muscular dystrophy gene, fukutin. FEBS Lett. 2001;489(2-3):192-196.

65. $\mathrm{Hu} \mathrm{H}$, et al. Conditional knockout of protein O-mannosyltransferase 2 reveals tissue-specific roles of O-mannosyl glycosylation in brain development. J Comp Neurol. 2011;519(7):1320-1337.

66 . Ackroyd M, et al. A new mouse model for dystroglycanopathies associated with mutations in FKRP. Paper presented at: World Muscle Society 2007; October 17-20, 2007; Sicily, Italy.

67. Satz JS, et al. Brain and eye malformations resembling Walker-Warburg syndrome are recapitulated in mice by dystroglycan deletion in the epiblast. J Neurosci. 2008;28(42):10567-10575.

68. Reed PW, Mathews KD, Mills KA, Bloch RJ. The sarcolemma in the Large(myd) mouse. Muscle
Nerve. 2004;30(5):585-595.

69. Chiba A, et al. Structures of sialylated O-linked oligosaccharides of bovine peripheral nerve alpha-dystroglycan. The role of a novel O-mannosyl-type oligosaccharide in the binding of alpha-dystroglycan with laminin. J Biol Chem. 1997;272(4):2156-2162.

70. Sasaki T, Yamada H, Matsumura K, Shimizu T, Kobata A, Endo T. Detection of O-mannosyl glycans in rabbit skeletal muscle alpha-dystroglycan. Biochim Biophys Acta. 1998;1425(3):599-606.

71. Smalheiser NR, Kim E. Purification of cranin, a laminin binding membrane protein. Identity with dystroglycan and reassessment of its carbohydrate moieties. J Biol Chem. 1995;270(25):15425-15433.

72. Ervasti JM, Campbell KP. A role for the dystrophin-glycoprotein complex as a transmembrane linker between laminin and actin. J Cell Biol. 1993; 122(4):809-823.

73. Gee SH, Blacher RW, Douville PJ, Provost PR, Yurchenco PD, Carbonetto S. Laminin-binding protein 120 from brain is closely related to the dystrophin-associated glycoprotein, dystroglycan, and binds with high affinity to the major heparin binding domain of laminin. J Biol Chem. 1993; 268(20):14972-14980.

74. Gee SH, Montanaro F, Lindenbaum MH, Carbonetto S. Dystroglycan-alpha, a dystrophin-associated glycoprotein, is a functional agrin receptor. Cell. 1994;77(5):675-686.

75. Smalheiser NR, Schwartz NB. Cranin: a lamininbinding protein of cell membranes. Proc Natl Acad Sci US A. 1987;84(18):6457-6461.

76. Yamada H, Shimizu T, Tanaka T, Campbell KP, Matsumura K. Dystroglycan is a binding protein of laminin and merosin in peripheral nerve. FEBS Lett. 1994;352(1):49-53.

77. Hayashi YK, et al. Selective deficiency of alpha-dystroglycan in Fukuyama-type congenital muscular dystrophy. Neurology. 2001;57(1):115-121.

78. Aravind L, Koonin EV. The fukutin protein family-predicted enzymes modifying cell-surface molecules. Curr Biol. 1999;9(22):R836-R837.

79. Xiong $\mathrm{H}$, et al. Molecular interaction between fukutin and POMGnT1 in the glycosylation pathway of alpha-dystroglycan. Biochem Biophys Res Commun. 2006;350(4):935-941.

80. Pereboev AV, Ahmed N, thi Man N, Morris GE. Epitopes in the interacting regions of beta-dystroglycan (PPxY motif) and dystrophin (WW domain). Biochim Biophys Acta. 2001;1527(1-2):54-60.

81. Beedle AM, Nienaber PM, Campbell KP. Fukutinrelated protein associates with the sarcolemmal dystrophin-glycoprotein complex. J Biol Chem. 2007; 282(23):16713-16717.

82. Miner JH, et al. The laminin alpha chains: expression, developmental transitions, and chromosomal locations of alpha1-5, identification of heterotrimeric laminins 8-11, and cloning of a novel alpha3 isoform. J Cell Biol. 1997;137(3):685-701. 